\title{
A jump-diffusion Libor model and its robust calibration
}

\author{
Denis Belomestny* and John Schoenmakers*
}

August 28, 2009

Keywords: jump-diffusion Libor models, calibration, stability, correlation structure

AMS 2000 Subject Classification: 60G51 62G20 60H05 60H10 90A09 91B28

\begin{abstract}
In this paper we propose a jump-diffusion Libor model with jumps in a high-dimensional space $\left(\mathbb{R}^{m}\right)$ and test a stable non-parametric calibration algorithm which takes into account a given local covariance structure. The algorithm returns smooth and simply structured Lévy densities, and penalizes the deviation from the Libor market model. In practice, the procedure is FFT based, thus fast, easy to implement, and yields good results, particularly in view of the severe ill-posedness of the underlying inverse problem.
\end{abstract}

\section{Introduction}

The calibration of financial models has become an important topic in financial engineering because of the need to price increasingly complex options consistent with prices of standard instruments liquidly traded in the market. The choice of an underlying model is crucial with respect to its statistical relevance on the one hand, and the possibility of calibrating it with ease on the other. In order to cover stylized facts in financial data such as implied volatility smiles and heavy tails, more complex models, i.e. models beyond Black-Scholes, are called for.

During the last decade Lévy-based models have drawn much attention, as these models are capable to describe complex but realistic behavior of financial time series. In particular, these models are well-suited to cover jumps, heavy tails, and to match implied volatility surfaces observed in stock and interest

*Partially supported by the Deutsche Forschungsgemeinschaft through SFB 649 "Economic Risk" and DFG Research Center Matheon "Mathematics for Key Technologies" in Berlin. Weierstrass Institute for Applied Analysis and Stochastics, Mohrenstr. 39, 10117 Berlin, \{belomest,schoenma\}@wias-berlin.de. 
rate markets. For modelling stock prices, pure jump Lévy processes were already proposed in Eberlein and Keller (1995), and Eberlein, Keller and Prause (1998). In Cont and Tankov (2003) regularized approaches for calibrating jump-diffusion stock price models were considered.

In the interest rate world the Libor market model developed by Brace, Gatarek and Musiela (1997), Jamshidian (1997), and Miltersen, Sandmann and Sondermann (1997), has become one of the most popular and advanced tools for modelling interest rates and interest rate derivatives. This in spite of a main drawback; the Libor market model cannot explain implied volatility surfaces typically observed in the cap markets. In order to handle this issue, different extensions involving processes with jumps have been proposed. Glasserman and Kou (2003) developed a jump-diffusion Libor model and proposed some explicit specifications of the driving jump processes. In Remark 2 we will discuss their approach in comparison with the present one. Eberlein and Özkan (2005) study the Libor model driven by a Lévy process. In this particular setting they give exponential integral representations for the Libor rates, and derive respective pricing formulas for caps. The most general framework for Libor models driven by jump measures is provided in Jamshidian (2001). Jamshidian's (2001) results will serve as baseplate for this article.

The central theme in this paper is a well structured jump-diffusion Libor model which allows for robust and efficient calibration. Special focus is put on modeling jumps with a tractable dependence structure which enables a feasible and robust calibration procedure later on. An effective treatment of this issue is missing in Glasserman and Kou (2003) and Eberlein and Özkan (2005) in fact. The starting point will be a given Libor market model with known deterministic volatility structure. For instance, this market model might be obtained from a calibration procedure involving at the money (ATM) caps, ATM swaptions, and/or a historically identified forward rate correlation structure. Meanwhile, calibration procedures for Libor market models are well studied in the literature (e.g. Brigo and Mercurio (2001) or Schoenmakers (2005)). Yet, our main goal is the development of a specific jump-diffusion Libor model which can be calibrated to the cap-strike matrix in a robust way and which is, in a sense, as near as possible to the given market model. In particular, this model will be furnished in such a way that the (local) covariance structure of the jumpdiffusion model coincides with the (local) covariance structure of the market model. We have three main reasons for doing so: (1) The price of a cap in a Libor market model does not depend on the (local) correlation structure of the forward Libors. However, this correlation structure may contain important information such as, for instance, prices of ATM swaptions. We therefore do not want to destroy this correlation structure as given by the input market model when calibrating the extended model to the cap(let)-strike volatility matrix. (2) The lack of smile behavior of the input market model, which is regarded as a rough intermediate approximation of a smile explaining jump-diffusion model, is considered to be a consequence of Gaussianity of the driving random forces (Wiener processes). So, loosely speaking, we want to perturb these forces to non-Gaussian ones by using jumps, while maintaining the (local) covariance 
structure of the given market model, hence the correlation structure implicitly. (3) Last but not least, by preserving the covariance structure we obtain a quite robust calibration procedure.

Many papers on calibration methods for Lévy based models focus on certain parametrizations of the underlying Lévy process. Since the characteristic triplet of a Lévy process is a priori an infinite-dimensional object, the parametric approach is always exposed to the problem of miss-specification. This is particularly the case when parametrizations are chosen just in view of generating different shapes of jump distributions, without further economical motivation. In this paper we therefore employ the nonparametric approach of Belomestny and Reiss (2004) which utilizes explicit inversion of a Fourier based pricing formula and a regularization in the spectral domain.

The outline of the paper is as follows. We recall in Section 2 the general arbitrage-free Libor framework developed in Jamshidian (2001). The covariance preserving jump-diffusion extension of the Libor market model is constructed in Section 3. In Section 4 we recap Fourier-based representations for Caplet prices in the spirit of Carr and Madan (1999), Glasserman and Merener (2003). The algorithm for calibrating to a full cap-strike matrix is developed in Section 5, and a real life calibration is carried out in Section 6. Technical details and derivations are given in the Appendix.

\section{General framework for Libor models with jumps}

Consider a fixed sequence of tenor dates $0=: T_{0}<T_{1}<T_{2}<\ldots T_{n}$, called a tenor structure, together with a sequence of so called day-count fractions $\delta_{i}:=T_{i+1}-T_{i}, \quad i=1, \ldots, n-1$. With respect to this tenor structure we consider zero coupon bond processes $B_{i}, i=1, \ldots, n$, where each $B_{i}$ lives on the interval $\left[0, T_{i}\right]$ and ends up with its face value $B_{i}\left(T_{i}\right)=1$. With respect to this bond system we deduce a system of forward rates, called Libor rates, which are defined by

$$
L_{i}(t):=\frac{1}{\delta_{i}}\left(\frac{B_{i}(t)}{B_{i+1}(t)}-1\right), \quad 0 \leq T_{i}, 1 \leq i \leq n-1 .
$$

Note that $L_{i}$ is the annualized effective forward rate to be contracted for at date $t$, for a loan over a forward period $\left[T_{i}, T_{i+1}\right]$. Based on this rate one has to pay at $T_{i+1}$ an interest amount of $\$ \delta_{i} L_{i}\left(T_{i}\right)$ on a $\$ 1$ notional. 


\subsection{Arbitrage free dynamics}

On a filtered measurable space $\left(\Omega, \mathcal{F}, \mathcal{F}_{t}\right)$ we consider a Libor model under the terminal measure $P_{n}$ within the following framework (Jamshidian (2001)),

$$
\begin{gathered}
\frac{d L_{i}}{L_{i-}}=-\sum_{j=i+1}^{n-1} \frac{\delta_{j} L_{j-}}{1+\delta_{j} L_{j-}} \eta_{i}^{\top} \eta_{j} d t+\eta_{i}^{\top} d W^{(n)} \\
-\int_{E} \nu^{(n)}(d t, d u) \psi_{i}(t, u)\left(\prod_{j=i+1}^{n-1}\left(1+\frac{\delta_{j} L_{j-} \psi_{j}(t, u)}{1+\delta_{j} L_{j-}}\right)-1\right) \\
+\int_{E} \psi_{i}(t, u)\left(\mu-\nu^{(n)}\right)(d t, d u), \quad i=1, \ldots, n-1,
\end{gathered}
$$

with $\omega \rightarrow \mu(d t, d u, \omega)$ being a random point measures on $\mathbb{R}_{+} \times E$, where $E$ is an abstract Lusin space, and $\nu^{(n)}(d t, d u, \omega)$ is the $\left(P_{n}, \mathcal{F}\right)$-compensator on $\mathbb{R}_{+} \times E$ of $\mu$. In $(1), W^{(n)}$ is a $d$-dimensional standard Brownian motion under $P_{n}$, and the filtration $\left(\mathcal{F}_{t}\right)_{t \geq 0}$ is assumed to contain the natural filtrations generated by $W^{(n)}$ and $\mu$, respectively. Further, $(\omega, t) \rightarrow \psi_{i}(t, \cdot, \omega)$ are predictable processes of functions on $E$, and $\eta_{i}$ are $d$-dimensional predictable column vector processes. The random measure $\mu$ is assumed to be of the form

$$
\mu=\sum_{n \geq 1} 1_{T_{n}(\omega)=t} \delta_{\left(t, \beta_{t}(\omega)\right)}(d t, d u),
$$

where $\beta$ is in general an optional process, and $T_{n}, n=1,2, .$. is a sequence of stopping times with disjoint graphs, i.e. $T_{n}(\omega) \neq T_{m}(\omega)$ for $n \neq m$.

The framework (1) may be cast into a somewhat different form. Let us consider a partition $E:=\bigcup_{k=1}^{m} E_{k}$, where $E_{1}, \ldots, E_{m}$ are Lusin spaces with $E_{k} \cap E_{l}=\varnothing$ for $k \neq l$, and define $\mu_{k}:=\left.\mu\right|_{E_{k}}, \psi_{i k}:=\left.\psi_{i}\right|_{E_{k}}, \nu_{k}^{(n)}:=\left.\nu^{(n)}\right|_{E_{k}}$, for $k=1, \ldots, m$. Then (1) becomes

$$
\begin{gathered}
\frac{d L_{i}}{L_{i-}}=-\sum_{j=i+1}^{n-1} \frac{\delta_{j} L_{j-}}{1+\delta_{j} L_{j-}} \eta_{i}^{\top} \eta_{j} d t+\eta_{i}^{\top} d W^{(n)} \\
-\sum_{k=1}^{m} \int_{E_{k}} \nu_{k}^{(n)}\left(d t, d u_{k}\right) \psi_{i k}\left(t, u_{k}\right)\left(\prod_{j=i+1}^{n-1}\left(1+\frac{\delta_{j} L_{j-} \psi_{j k}\left(t, u_{k}\right)}{1+\delta_{j} L_{j-}}\right)-1\right) \\
+\sum_{k=1}^{m} \int_{E_{k}} \psi_{i k}\left(t, u_{k}\right)\left(\mu_{k}-\nu_{k}^{(n)}\right)\left(d t, d u_{k}\right), \quad i=1, \ldots, n-1 .
\end{gathered}
$$

In particular, it easily follows that $\nu_{k}^{(n)}$ is the $P_{n}$-compensator of $\mu_{k}$ with respect to $\mathcal{F}$. Note that in general $E^{\mathcal{F}_{t}^{(k)}} \nu_{k}^{(n)}(\omega, d t, d u)$ is the compensator of $\mu_{k}$ with respect to the restricted filtration $\mathcal{F}_{t}^{(k)}:=\mathcal{F}_{t} \cap \sigma\{\mu([0, s] \times C): s \leq t, C \in$ $\left.\mathcal{B}\left(E_{k}\right)\right\}, t \geq 0$ (thus not $\nu_{k}^{(n)}$ ). As shown in Belomestny and Schoenmakers 
(2006), the representation (3) is in fact equivalent to (1), but somewhat more natural as it suggest the use of a system of $m$ point processes with phase space $\mathbb{R}_{+} \times \mathbb{R}$ as in the papers of Glasserman and Kou (2001), and Glasserman and Merener (2003).

Henceforth we consider in (1) only random point measures with finite activity, i.e., $\mu$ is of the form (2) and for each $t>0, \mu([0, t] \times E)<\infty$. In order to guarantee that the Libor processes $L_{i}$ are nonnegative we further require that $\psi_{i}>-1$ in $(1)$, and then set $\varphi_{i}:=\ln \left(\psi_{i}+1\right)$. Let $\left(s_{l}, u_{l}\right), l=1, \ldots, N_{t}$, denote the jumps of $\mu$ up to time $t$ for an $\omega \in \Omega$. Using the fact that at a jump time $s_{l}, \Delta L_{i}\left(s_{l}, \omega\right)=L_{i}\left(s_{l}-, \omega\right) \psi_{i}\left(s_{l}, u_{l}, \omega\right)=L_{i}\left(s_{l}-, \omega\right)\left(e^{\varphi_{i}\left(s_{l}, u_{l}, \omega\right)}-1\right)$, and so $L_{i}\left(s_{l}, \omega\right)=L_{i}\left(s_{l}-, \omega\right) e^{\varphi_{i}\left(s_{l}, u_{l}, \omega\right)}$, we obtain by the Ito-substitution rule for jump processes (with $\omega$ suppressed),

$$
\begin{aligned}
d \ln L_{i} & =\frac{1}{L_{i-}} d L_{i}-\frac{1}{2}\left|\eta_{i}\right|^{2} d t+d \sum_{l=1}^{N_{t}}\left(\varphi_{i}\left(s_{l}, u_{l}\right)-\psi_{i}\left(s_{l}, u_{l}\right)\right) \\
& =-\frac{1}{2}\left|\eta_{i}\right|^{2} d t-\sum_{j=i+1}^{n-1} \frac{\delta_{j} L_{j-}}{1+\delta_{j} L_{j-}} \eta_{i}^{\top} \eta_{j} d t+\eta_{i}^{\top} d W^{(n)} \\
& -\int_{E} \nu^{(n)}(d t, d u)\left(e^{\varphi_{i}(s, u)}-1\right) \prod_{j=i+1}^{n-1} \frac{1+\delta_{j} L_{j-} e^{\varphi_{j}(s, u)}}{1+\delta_{j} L_{j-}}+d \sum_{l=1}^{N_{t}} \varphi_{i}\left(s_{l}, u_{l}\right) .
\end{aligned}
$$

The logarithmic analog of (3) directly follows from (4),

$$
\begin{aligned}
d \ln L_{i} & =-\frac{1}{2}\left|\eta_{i}\right|^{2} d t-\sum_{j=i+1}^{n-1} \frac{\delta_{j} L_{j-}}{1+\delta_{j} L_{j-}} \eta_{i}^{\top} \eta_{j} d t+\eta_{i}^{\top} d W^{(n)} \\
& -\sum_{k=1}^{m} \int_{E_{k}} \nu_{k}^{(n)}\left(d t, d u_{k}\right)\left(e^{\varphi_{i k}\left(s, u_{k}\right)}-1\right) \prod_{j=i+1}^{n-1} \frac{1+\delta_{j} L_{j-} e^{\varphi_{j k}\left(s, u_{k}\right)}}{1+\delta_{j} L_{j-}} \\
& +d \sum_{k=1}^{m} \sum_{l=1}^{N_{t}^{(k)}} \varphi_{i k}\left(s_{l}^{(k)}, u_{l}^{(k)}\right),
\end{aligned}
$$

with $\varphi_{i k}:=\ln \left(\psi_{i k}+1\right)$ and $\left(s_{l}^{(k)}, u_{l}^{(k)}\right), l=1, \ldots, N_{t}^{(k)}$, denoting the jumps of $\mu_{k}$ up to time $t$. The logarithmic representation (4) (or equivalently (5)) will be the basic framework for our purposes.

\section{Jump diffusion extension of a Libor market model}

We first specialize to a jump-diffusion Libor model which is driven by a Poisson random measure with marks in some multi-dimensional space. 


\subsection{Poisson driven multi-dimensional jumps}

On the space $\mathbb{R}_{+} \times E$, where now $E:=\mathbb{R}^{m}$, we consider a jump measure $\mu$ with deterministic $\left(P_{n}, \mathcal{F}\right)$-compensator of the form

$$
\nu^{(n)}\left(d t, d u_{1}, \ldots, d u_{m}\right):=\lambda(t) p_{1}\left(d u_{1}\right) \cdots p_{m}\left(d u_{m}\right) d t=: \lambda(t) p(d u) d t,
$$

with $p_{i}\left(d u_{i}\right)$ being Borel probability measures on $\mathbb{R}$, and $\lambda$ a non-negative locally integrable Borel function on $[0, \infty)$. As a consequence, the jump times of the measure $\mu$ are Poisson distributed with locally finite intensity measure $\lambda(t) d t$, and in particular, given a jump time $\tau, \mu(\{\tau\}, \omega)=\delta_{\left(\tau, u_{\tau}\right)}(\omega)$, where the jump $u_{\tau} \in \mathbb{R}^{m}$ is sampled by drawing it's components $u_{i}$ independently from the measures $p_{i}\left(d u_{i}\right), i=1, \ldots, m$, respectively. Furthermore, by this construction, the $P_{n}$ standard Brownian motion $W^{(n)}$ is independent of $\mu$.

\subsection{Extending the Libor market model}

Within the framework in Section 2 we now introduce a jump-diffusion Libor model using the jump measure constructed in Section 3.1, that in a sense can be seen as an extension or perturbation of a (given) Libor market model. Let $\gamma_{i}(t) \in \mathbb{R}^{d}$ be the (given) deterministic volatility structure of the market model, resulting for instance from some standard calibration procedure to ATM caps and ATM swaptions or historical data. To exclude local redundancies we assume that the matrix $\left(\gamma_{i, l}(t)\right)_{1 \leq i<n, 1 \leq l \leq d}$ has full rank $d$ for all $t$. In connection with the Poisson measure introduced in Section 3.1 we consider deterministic vector functions $\beta_{i}(t) \in \mathbb{R}^{m}, i=1, \ldots, n-1$, take a sequence of constants $r_{i}$ with $0 \leq r_{i} \leq 1$, and then set

$$
\eta_{i}:=\sqrt{1-r_{i}^{2}} \gamma_{i}, \quad \varphi_{i}(t, u):=r_{i} u^{\top} \beta_{i}(t)
$$

in (4) to yield,

$$
\begin{array}{r}
d \ln L_{i}=-\frac{1}{2}\left(1-r_{i}^{2}\right)\left|\gamma_{i}\right|^{2} d t-\sum_{j=i+1}^{n-1} \frac{\delta_{j} L_{j-}}{1+\delta_{j} L_{j-}} \sqrt{\left(1-r_{i}^{2}\right)\left(1-r_{j}^{2}\right)} \gamma_{i}^{\top} \gamma_{j} d t \\
+\sqrt{1-r_{i}^{2}} \gamma_{i}^{\top} d W^{(n)}+r_{i} d \sum_{l=1}^{N_{t}} u_{l}^{\top} \beta_{i}\left(s_{l}\right) \\
-\lambda(t) d t \int_{\mathbb{R}^{m}}\left(\exp \left(r_{i} u^{\top} \beta_{i}\right)-1\right) p(d u) \prod_{j=i+1}^{n-1} \frac{1+\delta_{j} L_{j-} \exp \left(r_{j} u^{\top} \beta_{j}\right)}{1+\delta_{j} L_{j-}} .
\end{array}
$$

Note that in (7) the market model is retrieved by taking $r_{i} \equiv 0$, and so, for small $r_{i},(7)$ may be seen as a jump diffusion perturbation of the Libor market model. 


\subsection{The jump drift of $\ln L_{i}$ under $P_{n}$}

Let us consider the third term in (7), i.e. the "jump drift" of $\ln L_{i}$ under the terminal measure $P_{n}$. The computation of this term is of particular importance, for example, in a Monte Carlo simulation of the model. For a fixed time $t>0$ we consider the expression

$$
(*):=\int_{\mathbb{R}^{m}} p(d u)\left(\exp \left(r_{i} u^{\top} \beta_{i}(t)-1\right) \prod_{j=i+1}^{n-1}\left[1+\delta_{j} L_{j-}(t) \exp \left(r_{j} u^{\top} \beta_{j}(t)\right)\right] .\right.
$$

Using the abbreviation $x_{j}:=\delta_{j} L_{j-}(t) \exp \left(r_{j} u^{\top} \beta_{j}(t)\right)$, the product in (8) my be expanded as

$$
\begin{aligned}
\prod_{j=i+1}^{n-1}\left(1+x_{j}\right) & =1+\sum_{i<j<n} x_{j}+\sum_{i<j_{1}<j_{2}<n} x_{j_{1}} x_{j_{2}} \\
& +\sum_{i<j_{1}<j_{2}<j_{3}<n} x_{j_{1}} x_{j_{2}} x_{j_{3}}+\ldots+x_{i+1} \cdots x_{n-1} .
\end{aligned}
$$

Let us take a generic term of degree $1 \leq d<n-i$ (with $t$ suppressed),

$$
x_{j_{1}} \cdots x_{j_{d}}=\delta_{j_{1}} L_{j_{1}-} \cdots \delta_{j_{d}} L_{j_{d}-} \exp \left(r_{j_{1}} u^{\top} \beta_{j_{1}}\right) \cdots \exp \left(r_{j_{d}} u^{\top} \beta_{j_{d}}\right) \text {, }
$$

for $i<j_{1}<j_{2}<\cdots<j_{d}<n$, and observe that

$$
\begin{aligned}
& \int_{\mathbb{R}^{m}} p(d u) e^{r_{i} u^{\top} \beta_{i}} \exp \left(r_{j_{1}} u^{\top} \beta_{j_{1}}\right) \cdots \exp \left(r_{j_{d}} u^{\top} \beta_{j_{d}}\right) \\
& =\int_{\mathbb{R}^{m}} p(d u) \exp \left[u^{\top}\left(r_{i} \beta_{i}+r_{j_{1}} \beta_{j_{1}}+\cdots+r_{j_{d}} \beta_{j_{d}}\right)\right] \\
& =\prod_{l=1}^{m} \int_{\mathbb{R}} p_{l}\left(d u_{l}\right) \exp \left[u_{l}\left(r_{i} \beta_{i l}+r_{j_{1}} \beta_{j_{1} l}+\cdots+r_{j_{d}} \beta_{j_{d} l}\right)\right] \\
& =\prod_{l=1}^{m} \phi_{p_{l}}\left(-\mathfrak{i} r_{i} \beta_{i l}-\mathfrak{i} r_{j_{1}} \beta_{j_{1} l} \cdots-\mathfrak{i} r_{j_{d}} \beta_{j_{d} l}\right),
\end{aligned}
$$

with $\phi_{p_{l}}$ being the characteristic function of $p_{l}$. Note that the existence of $\phi_{p_{l}}(z)$ in some ball $\{z \in \mathbb{C}:|z|<A\}$ has to be assumed. By analogue computations 
and collecting terms we thus obtain

$$
\begin{gathered}
(*)=-1+\prod_{l=1}^{m} \phi_{p_{l}}\left(-\mathfrak{i} r_{i} \beta_{i l}\right)+ \\
\sum_{d=1}^{n-1-i} \sum_{i<j_{1}<j_{2}<\cdots<j_{d}<n} \delta_{j_{1}} L_{j_{1}-} \cdots \delta_{j_{d}} L_{j_{d}-} \times \\
\times\left[\prod_{l=1}^{m} \phi_{p_{l}}\left(-\mathfrak{i} r_{i} \beta_{i l}-\mathfrak{i} r_{j_{1}} \beta_{j_{1} l} \cdots-\mathfrak{i} r_{j_{d}} \beta_{j_{d} l}\right)-\prod_{l=1}^{m} \phi_{p_{l}}\left(-\mathfrak{i} r_{j_{1}} \beta_{j_{1} l} \cdots-\mathfrak{i} r_{j_{d}} \beta_{j_{d} l}\right)\right] \\
=: \varrho_{i}^{p, r, \beta}+\sum_{d=1}^{n-1-i} \sum_{i<j_{1}<j_{2}<\cdots<j_{d}<n} \delta_{j_{1}} L_{j_{1}-} \cdots \delta_{j_{d}} L_{j_{d}-} \varrho_{i ; j_{1}, \ldots, j_{d}}^{p, r, \beta}
\end{gathered}
$$

Once the model inputs $r_{i}$, jump loadings $t \rightarrow \beta_{i}(t), 1 \leq i<n$, and jump component measures $p_{l}$ with characteristic functions $\phi_{p_{l}}, 1 \leq l \leq m$, are calibrated or simply given, the real valued functions $t \rightarrow \varrho_{i}^{p, r, \beta}(t), t \rightarrow \varrho_{i ; j_{1}, \ldots, j_{d}}^{p, r, \beta}(t), 1 \leq i<n$, $i<j_{1}<j_{2}<\cdots<j_{d}<n$, can be computed in closed form and, in principle, can even be stored outside the Monte Carlo simulator. Thus considering these functions as given, the simulation of $\ln L_{i}$ in the terminal measure may be carried out straightforwardly via the formula

$$
\begin{aligned}
d \ln L_{i}= & -\frac{1}{2}\left(1-r_{i}^{2}\right)\left|\gamma_{i}\right|^{2} d t-\sum_{j=i+1}^{n-1} \frac{\delta_{j} L_{j-}}{1+\delta_{j} L_{j-}} \sqrt{\left(1-r_{i}^{2}\right)\left(1-r_{j}^{2}\right)} \gamma_{i}^{\top} \gamma_{j} d t \\
& +\sqrt{1-r_{i}^{2}} \gamma_{i}^{\top} d W^{(n)}+r_{i} d \sum_{l=1}^{N_{t}} u_{l}^{\top} \beta_{i}\left(s_{l}\right) \\
& -\prod_{j=i+1}^{n-1}\left(1+\delta_{j} L_{j-}\right)^{-1} \lambda(t) d t\left[\varrho_{i}^{p, r, \beta}(t)+\right. \\
& \left.+\sum_{d=1}^{n-1-i} \sum_{i<j_{1}<j_{2}<\cdots<j_{d}<n} \delta_{j_{1}} L_{j_{1}-} \cdots \delta_{j_{d}} L_{j_{d}-\varrho_{i ; j_{1}, \ldots, j_{d}}^{p, r, \beta}}(t)\right] .
\end{aligned}
$$

We underline that the structure of the dynamics (9), hence the feasibility of standard Monte Carlo simulation of every forward Libor in the terminal measure, is a consequence of our model design in Sections 3.1 and 3.2. In particular it is due to the special product structure of the generally high dimensional jump measure $p$ and the linear structure of the log-Libor factor loadings (6).

Remark 1 Based on (9) we may consider different Libor model approximations. For example we may freeze $L_{j-}$ at zero (see Glasserman and Merener (2003)), hence replace $L_{j-}$ with $L_{j}(0)$ in (9). As an alternative, if the $r_{i}$ are small enough and the magnitudes of $\delta_{j} L_{j}$ are small enough as well, one could drop in (9) the terms of order $\left(\delta_{j} L_{j}\right)^{2}$ and higher. Of course, any such attempt needs careful investigation which is considered beyond the scope of this article. 
For related approximations in the context of the standard Libor market model, see for instance Kurbanmuradov, Sabelfeld and Schoenmakers (2002).

Remark 2 It is interesting to consider and compare our setup with the one Glasserman and Kou (2003). A common goal of our approach and the one in Glasserman \& Kou (2003) is specializing from a general Libor framework with jumps to a subclass of models which are tractable from an analytic point of view and from a simulation point of view. There is an essential difference in the way of specializing however. In particular, the way of modeling the dependence structure for different forwards is different in Glasserman \& Kou (2003): Glasserman \& Kou (2003) deal with a set of (possibly correlated) marked point processes with a simple state space $([0, \infty))$. The jumps of a forward Libor $L_{j}$ are then due to the aggregated effect of a subset $I_{j}$ of these point processes to which $L_{j}$ is sensitive via a simple sensitivity function $u \rightarrow u-1$ for the mark $u \in[0, \infty)$. What is missing in Glasserman \& Kou (2003) is in fact a tractable framework for the dependence structure of the different one-dimensional point processes. Of course, taking just a single point process on $[0, \infty)$ would lead to perfectly correlated jumps of all forward Libors which may be too restrictive in practice.

In our approach we have only one (compound Poisson) point process, but with a richer state space $\left(\mathbb{R}^{m}\right)$, and a sensitivity function of the form $\psi_{i}=$ $\exp \left[u^{\top} \beta_{i}\right]-1$ for the mark $u \in \mathbb{R}^{m}$. Given a jump time $\tau$ the components of $u$ are independently sampled and coupled via the vector $\beta_{i}$ specific for $L_{i}$ which determines the dependence structure of the Libor jumps. In particular when a jump time occurs all forwards jump in a correlated way determined by the vectors $\beta_{i}$. So in our setup the jump dependencies are naturally structured which, as we will see, allows for a feasible calibration procedure. Furthermore, the special choice of the sensitivity function $\psi_{i}$ leads to more analytical tractability.

\subsection{Dynamics of $L_{i}$ under $P_{i+1}$}

We now consider for $i=1, \ldots, n-1$ the dynamics of $L_{i}$ under $P_{i+1}$. From (7) we see that the logarithm of the last Libor rate $L_{n-1}$ has the following simple dynamics in the $P_{n}$ measure,

$$
\begin{gathered}
d \ln L_{n-1}=-\frac{1}{2}\left(1-r_{n-1}^{2}\right)\left|\gamma_{n-1}\right|^{2} d t+\sqrt{1-r_{n-1}^{2}} \gamma_{n-1}^{\top} d W^{(n)} \\
+r_{n-1} d \sum_{l=1}^{N_{t}} u_{l}^{\top} \beta_{n-1}\left(s_{l}\right)-\lambda(t) d t \int_{\mathbb{R}^{m}}\left(\exp \left(r_{n-1} u^{\top} \beta_{n-1}\right)-1\right) p(d u),
\end{gathered}
$$

and thus belongs to the class of additive models, i.e., the process $X_{n-1}(t):=$ $\ln L_{n-1}(t)-\ln L_{n-1}(0)$ has independent increments. By using Lemma 3 below for instance, we can derive straightforwardly the characteristic function of 


$$
\begin{aligned}
X_{n-1}(t), & \\
\Phi_{n}(z ; t):= & E_{P_{n}} \exp \left[\mathfrak{i} z X_{n-1}(t)\right]=\exp \left[\psi_{n}(z ; t)\right] \\
\psi_{n}(z ; t) & :=-\frac{z^{2}}{2}\left(1-r_{n-1}^{2}\right) \int_{0}^{t}\left|\gamma_{n-1}(s)\right|^{2} d s-\mathfrak{i} z \int_{0}^{t}\left[\frac{1}{2}\left(1-r_{n-1}^{2}\right)\left|\gamma_{n-1}(s)\right|^{2} d s+\right. \\
& \left.\lambda(s) d s \int_{\mathbb{R}^{m}}\left(\exp \left(r_{n-1} u^{\top} \beta_{n-1}(s)\right)-1\right) p(d u)\right] \\
& +\int_{0}^{t} \lambda(s) d s \int_{\mathbb{R}^{m}}\left(e^{\mathfrak{i} z r_{n-1} u^{\top} \beta_{n-1}(s)}-1\right) p(d u)
\end{aligned}
$$

For $1 \leq i<n-1$ the dynamics of of $L_{i}$ under $P_{i+1}$ is more complicated. By the fact that $L_{i}$ is a martingale under $P_{i+1}$ we observe from the general framework (1) that

$$
\frac{d L_{i}}{L_{i-}}=: \eta_{i}^{\top} d W^{(i+1)}+\int_{E} \psi_{i}(t, u)\left(\mu-\nu^{(i+1)}\right)(d t, d u)
$$

where

$$
d W^{(i+1)}=-\sum_{j=i+1}^{n-1} \frac{\delta_{j} L_{j-}}{1+\delta_{j} L_{j-}} \eta_{j} d t+d W^{(n)}
$$

is a standard Brownian motion under $P_{i+1}$, and

$$
\nu^{(i+1)}(d t, d u)=\nu^{(n)}(d t, d u) \prod_{j=i+1}^{n-1}\left(1+\frac{\delta_{j} L_{j-} \psi_{j}(t, u)}{1+\delta_{j} L_{j-}}\right)
$$

is the compensator process of $\mu$ under the measure $P_{i+1}$. For the more specialized setup introduced in this section, which is based on $(6),(14)$ reads

$$
\nu^{(i+1)}(d t, d u)=\lambda(t) p(d u) d t \prod_{j=i+1}^{n-1} \frac{1+\delta_{j} L_{j-} \exp \left(r_{j} u^{\top} \beta_{j}\right)}{1+\delta_{j} L_{j-}},
$$

and (13) reads

$$
\frac{d L_{i}}{L_{i-}}=\sqrt{1-r_{i}^{2}} \gamma_{i}^{\top} d W^{(i+1)}+\int_{\mathbb{R}^{m}}\left(e^{r_{i} u^{\top} \beta_{i}(t)}-1\right)\left(\mu-\nu^{(i+1)}\right)(d t, d u),
$$

$i=1, \ldots, n-1$. The logarithmic version of (16) is seen from (7) to be

$$
\begin{gathered}
d \ln L_{i}=-\frac{1}{2}\left(1-r_{i}^{2}\right)\left|\gamma_{i}\right|^{2} d t+\sqrt{1-r_{i}^{2}} \gamma_{i}^{\top} d W^{(i+1)} \\
+r_{i} d \sum_{l=1}^{N_{t}} u_{l}^{\top} \beta_{i}\left(s_{l}\right)-\int_{\mathbb{R}^{m}}\left(\exp \left(r_{i} u^{\top} \beta_{i}\right)-1\right) \nu^{(i+1)}(d t, d u) .
\end{gathered}
$$

In particular, for $i<n-1$ the compensator (15) is non-deterministic in the present setup and, as a consequence, $\ln L_{i}$ is generally not additive under $P_{i+1}$ for $i<n-1$. However, by freezing in (15) the Libor terms, i.e. replacing $L_{i-}$ by $L_{i-}(0)$, we may obtain a deterministic approximative compensator and so an additive approximation of $\ln L_{i}$ under $P_{i+1}$. 


\subsection{Preserving the local covariance structure}

We recall the following standard lemma proved in Belomestny and Schoenmakers (2006).

Lemma 3 If $J(t)=\sum_{l=1}^{N_{t}} \varphi\left(s_{l}, u_{l}\right)$ is a compound Poisson process in $\mathbb{R}^{q}$ with jump intensity $\lambda(t) d t$, independent jumps in a measurable space $E$ with probability measure $p(d u)$, and $\varphi: \mathbb{R}_{+} \times E \rightarrow \mathbb{R}^{q}$ is deterministic, then (i) the characteristic function of $J(t)$ is given by

$$
E e^{\mathfrak{i} z^{\top} J(t)}=\exp \left[\int_{0}^{t} \lambda(s) d s \int_{E}\left(e^{\mathfrak{i} z^{\top} \varphi(s, u)}-1\right) p(d u)\right], \quad z \in \mathbb{R}^{q},
$$

and (ii) for the expectation and covariance structure of $J(t)$ we have

$$
\begin{aligned}
E J_{l}(t) & =\int_{0}^{t} \lambda(s) d s \int_{E} \varphi_{l}(s, u) p(d u), \\
\operatorname{Cov}\left(J_{l}(t), J_{l^{\prime}}(t)\right) & =\int_{0}^{t} \lambda(s) d s \int_{E} \varphi_{l}(s, u) \varphi_{l^{\prime}}(s, u) p(d u), \quad 1 \leq l, l^{\prime} \leq q .
\end{aligned}
$$

Let us now write the integrated random term in (7) as

$$
\begin{aligned}
\xi_{i}(t) & :=\sqrt{1-r_{i}^{2}} \int_{0}^{t} \gamma_{i}^{\top} d W^{(n)}+r_{i} \sum_{l=1}^{N_{t}} u_{l}^{\top} \beta_{i}\left(s_{l}\right) \\
& =: \sqrt{1-r_{i}^{2}} \xi_{i}^{D}(t)+r_{i} \xi_{i}^{J}(t) .
\end{aligned}
$$

By Lemma 3 the characteristic function of the jump process $\xi^{J}$ is then given by

$$
E e^{\mathfrak{i} z^{\top} \xi^{J}(t)}=\exp \left[\int_{0}^{t} \lambda(s) d s\left(\phi_{p}\left(\sum_{j=1}^{n-1} z_{j} \beta_{j}(s)\right)-1\right)\right],
$$

with $\phi_{p}(y):=\int p(d u) \exp \left[\mathfrak{i} u^{\top} y\right], y \in \mathbb{R}^{m}$, being the characteristic function of $p$. For the covariance matrix Lemma 3 yields

$$
\begin{aligned}
\operatorname{Cov}\left(\xi_{i}^{J}(t), \xi_{j}^{J}(t)\right) & =\int_{0}^{t} \lambda(s) d s \int_{\mathbb{R}^{m}} \beta_{i}^{\top}(s) u u^{\top} \beta_{j}(s) p(d u) \\
& =: \int_{0}^{t} \lambda(s) d s \beta_{i}^{\top}(s) \Sigma \beta_{j}(s)
\end{aligned}
$$

with $\Sigma_{k l}:=\int u_{k} u_{l} p(d u)$ being the cross moments of jump components $u_{k}$ and $u_{l}$. Since the Brownian motion and the jumps are independent we have for the local covariance (actually the predictable compensator) of $\xi_{i}$ and $\xi_{j}$ in (18),

$$
\operatorname{Cov}\left(d \xi_{i}(t), d \xi_{j}(t)\right)=\sqrt{\left(1-r_{i}^{2}\right)\left(1-r_{j}^{2}\right)} \gamma_{i}^{\top}(t) \gamma_{j}(t)+r_{i} r_{j} \lambda(t) \beta_{i}^{\top}(t) \Sigma \beta_{j}(t) d t
$$


Our main idea is to consider jump diffusion extensions of a (given) pure Libor market model which preserve the (given) local covariance structure of the market model. To this aim we consider in (7) the case where $r: \equiv r_{i}$ for all $i$. Then (19) yields

$$
\operatorname{Cov}\left(d \xi_{i}, d \xi_{j}\right)=\left(1-r^{2}\right) \gamma_{i}^{\top} \gamma_{j}+r^{2} \lambda \beta_{i}^{\top} \Sigma \beta_{j} d t
$$

We then assume $\beta_{j}=A \gamma_{j}$ for some $m \times d$ matrix $A$ which gives

$$
\operatorname{Cov}\left(d \xi_{i}, d \xi_{j}\right)=\gamma_{i}^{\top}\left(I-r^{2} I+r^{2} \lambda A^{\top} \Sigma A\right) \gamma_{j} / d t
$$

The requirement that the local covariances (19) coincide with the local covariances of the market model now leads to the condition

$$
\lambda A^{\top} \Sigma A=I_{d},
$$

and in particular $m \geq d$. Since $\Sigma$ is (time independent) positive definite there is a unique positive symmetric $m \times m$ matrix $C$ such that $\Sigma=C^{2}$. Then for any column-orthogonal $m \times d$ matrix $Q$ we have a solution

$$
A=\lambda^{-1 / 2} C^{-1} Q \text {. }
$$

Note that in general $Q$ and $\lambda$ may depend on $t$. Without loss of generality (i.e. without affecting the input Libor market model) we may assume that the $(n-1) \times d$ matrix $\left(\gamma_{j, r}\right)$ is an upper triangular matrix in the sense

$$
\gamma_{n-j, l}=0 \quad \text { for } \quad 1 \leq l<d-j+1, \quad j=1, \ldots, d .
$$

We assume (for technical reasons in fact) that the $(n-1) \times m$ matrix $\left(\beta_{j, r}\right)$ is also an upper triangular matrix,

$$
\beta_{n-j, l}=\sum_{r=1}^{d} A_{l, r} \gamma_{n-j, r}=0, \quad \text { for } \quad 1 \leq l<m-j+1, \quad j=1, \ldots, m .
$$

In particular this entails that the jumps of $L_{n-1}$ are driven by a single jump measure. We will achieve (20) by the additional requirement $m=d$ (dimension of the jump space equal to the number of Brownian motions) and by taking the orthogonal matrix $Q$ such that $C^{-1} Q$, hence $A$, is a lower triangular (square) matrix with positive diagonal elements. Thus, $A$ is uniquely determined by

$$
A A^{\top}=\lambda^{-1} \Sigma^{-1}, \quad A \text { is lower triangular with positive diagonal }
$$

(hence time independent). As a further specialization we take $\lambda$ to be time independent. Note that $u^{\top} \beta_{i}=(D u)^{\top} D^{-1} \beta_{i}$ for any regular diagonal matrix $D$. So, multiplication of all jump random variables with an arbitrary factor and respective components of $\beta_{i}$ with this factor's inverse yields the same model. Therefore, without any restriction we may fix the jump variances $\alpha_{k}$ defined as

$$
\begin{aligned}
\alpha_{k} & :=\int u_{k}^{2} p_{k}\left(d u_{k}\right)-\kappa_{k}^{2}, \quad \text { where } \\
\kappa_{k} & :=\int u_{k} p_{k}\left(d u_{k}\right)
\end{aligned}
$$


is the mean of the $k$ th jump component, as we like. As a convenient choice we take them all equal, i.e. we set $\alpha_{k} \equiv: \alpha, k=1, \ldots, m$. Next, we will choose $\alpha$ such that $\|A\|_{F}:=\sqrt{\sum_{k, l=1}^{m}\left|A_{k l}\right|^{2}}=\sqrt{m}=\left\|I_{m}\right\|_{F}$, which is equivalent to

$$
\left\|C^{-1}\right\|_{F}^{2}=\sum_{k=1}^{m} \frac{1}{\lambda_{k}^{\Sigma}}=\lambda m,
$$

where $\lambda_{k}^{\Sigma}, k=1, \ldots, m$, denote the eigenvalues of $\Sigma$. Then by the result of Appendix 7.3 it follows that (24) is equivalent to

$$
\alpha \lambda=\frac{\alpha+\frac{m-1}{m} \sum_{p=1}^{m} \kappa_{p}^{2}}{\alpha+\sum_{p=1}^{m} \kappa_{p}^{2}} .
$$

It is easy to show that this quadratic equation in $\alpha$ has one positive and one negative solution, and that for large $m$ the positive solution $\alpha_{+} \approx 1 / \lambda$. We therefore set

$$
\alpha:=\frac{1}{\lambda} \equiv \alpha_{k}, \quad k=1, \ldots, m .
$$

By denoting the columns of $C$ by $c_{k}, k=1, \ldots, m$, we have for $k, l=1, \ldots, m$, $c_{k}^{\top} c_{l}=e_{k}^{\top} C^{2} e_{l}=\Sigma_{k l}=\alpha_{k} \delta_{k l}+\kappa_{k} \kappa_{l}$. We so have in particular $\beta_{n-1, l}(s) \equiv 0$ for $1 \leq l<m$, and

$$
\begin{aligned}
\beta_{n-1, m}(s) & =A_{m, m} \gamma_{n-1, m}(s)=\lambda^{-1 / 2}\left(e_{m}^{\top} C^{2} e_{m}\right)^{-1} \gamma_{n-1, m}(s) \\
& =\frac{\gamma_{n-1, m}(s)}{\sqrt{\lambda\left(\alpha+\kappa_{m}^{2}\right)}}=\frac{\gamma_{n-1, m}(s)}{\sqrt{1+\lambda \kappa_{m}^{2}}} .
\end{aligned}
$$

Hence the dynamics of $\ln L_{n-1}$ is driven by a single jump variable $u_{m}$ under a jump distribution $p_{m}$, with mean $\kappa_{m}$ and variance $\lambda^{-1}$.

Discussion Before turning to the calibration let us summarize the main features of our model and describe the main ideas behind the calibration procedure. For any $i=1, \ldots, n-1$ the main parameters of the model for $L_{i}$ under $P_{n}$ in (7) are

1. Vector functions $\gamma_{j}(t), j=i, \ldots, n-1$ which determine the correlation structure of the Libor Market Model we start with.

2. Vector functions $\beta_{j}(t), j=i, \ldots, n-1$ that scale the jumps and are related to $\gamma_{j}(t)$ as in $(20)$.

3. The intensity $\lambda$ of the driving Poisson process $N_{t}$.

4. The weight $r$ which determines the balance between diffusion and jump components in $L_{i}$. 


\section{Jump component measures $p_{1}, \ldots, p_{m}$.}

Note that while the first four components are of parametric nature (we parameterize the functions $\gamma_{i}(t)$ later on), the measures $p_{i}$ are the nonparametric ingredients of the model. In the first pre-calibration step we fit a Libor market model using at-the-money caplets and at-the-money swaptions for example. This gives us vector functions $\gamma_{1}(t), \ldots, \gamma_{n-1}(t)$, and then $\beta_{1}(t), \ldots, \beta_{n-1}(t)$ up to parameters $\alpha$ and $\kappa_{1}(t), \ldots, \kappa_{m}(t)$ defined in (22) and (23) respectively. Note that due to the covariance constraint (20) we avoid a complete calibration of the $\beta$ 's that renders the procedure more stable. Finally, we calibrate $\lambda$ (which equals $1 / \alpha$ ), $r$, and $p_{1}, \ldots, p_{m}$ (and so the $\kappa$ 's) to the whole matrix of caplet volatilities. By imposing a triangle structure on the $\gamma$ 's and $\beta$ 's it will be achieved that the dynamic of $L_{i}$ depends on $p_{i}, \ldots, p_{m}$ only. This in turn makes it possible to develop a calibration algorithm which starts with $i=n-1$

and proceeds backwardly in such a way that at the time $i<n-1$ all measures $p_{i+1}, \ldots, p_{m}$ are already estimated and it remains to determine $p_{i}$.

\section{Pricing caplets}

A caplet for the period $\left[T_{j}, T_{j+1}\right]$ with strike $K$ is an option which pays $\left(L_{j}\left(T_{j}\right)-\right.$ $K)^{+} \delta_{j}$ at time $T_{j+1}$, where $1 \leq j<n$. It is well-known that under the $T_{j+1}$ - forward measure the caplet price has the following simple representation. Writing $E_{j+1}$ for the expectation under this measure, we have

$$
C_{j}(K)=B_{j+1}(0) E_{j+1}\left[\left(L_{j}\left(T_{j}\right)-K\right)^{+} \delta_{j}\right]
$$

for price of the $j$-th caplet at time zero. Thus the $j$-th caplet price is determined by the dynamics of $L_{j}$ under $P_{j+1}$ only. We now recall the FFT pricing method of Carr and Madan, which basically goes as follows. It turns out natural to transform for a fixed $j$ the strike variable into a log-forward moneyness variable defined by

$$
v:=\ln \frac{K}{L_{j}(0)} .
$$

In terms of log-forward moneyness the $j$-th caplet price is then given by

$$
\mathcal{C}_{j}(v):=\delta_{j} B_{j+1}(0) L_{j}(0) E_{j+1}\left[\left(e^{X_{j}\left(T_{j}\right)}-e^{v}\right)^{+}\right],
$$

where $X_{j}(t):=\ln L_{j}(t)-\ln L_{j}(0)$. We further introduce an auxiliary function

$$
\begin{aligned}
\mathcal{O}_{j}(v) & :=\delta_{j}^{-1} B_{j+1}^{-1}(0) L_{j}^{-1}(0) \mathcal{C}_{j}(v)-\left(1-e^{v}\right)^{+} \\
& =E_{j+1}\left(e^{X_{j}\left(T_{j}\right)}-e^{v}\right)^{+}-\left(1-e^{v}\right)^{+} \\
& =1_{v \geq 0} E_{j+1}\left(e^{X_{j}\left(T_{j}\right)}-e^{v}\right)^{+}+1_{v \leq 0} E_{j+1}\left(e^{v}-e^{X_{j}\left(T_{j}\right)}\right)^{+}
\end{aligned}
$$

where the third expression is basically due to the put-call parity and follows from the identity $(a-b)^{+}=a-b+(b-a)^{+}$and the fact $E_{j+1} e^{X_{j}\left(T_{j}\right)}=1$. 
In the Appendix we derive further characteristic properties of the function $\mathcal{O}_{j}$. The Fourier transform of $\mathcal{O}_{j}$ is given by

$$
\mathfrak{F}\left\{\mathcal{O}_{j}\right\}(z)=\int_{-\infty}^{\infty} \mathcal{O}_{j}(v) e^{\mathfrak{i} v z} d v=\frac{1-\Phi_{j+1}\left(z-\mathfrak{i} ; T_{j}\right)}{z(z-\mathfrak{i})} .
$$

This can be proved via a straightforward reformulation of a similar result in Cont and Tankov (2003) in the context of jump-diffusion asset model (see Belomestny and Schoenmakers (2006)).

Most importantly, if the characteristic function of $X_{j}\left(T_{j}\right)$ is explicitly given, for example by (11), and (12) in the case $j=n-1$, we obtain an analytical caplet pricing formula via Fourier inversion,

$$
\begin{aligned}
C_{j}(K)= & \delta_{j} B_{j+1}(0)\left(L_{j}(0)-K\right)^{+}+ \\
& \frac{\delta_{j} B_{j+1}(0) L_{j}(0)}{2 \pi} \int_{-\infty}^{\infty} \frac{1-\Phi_{j+1}\left(z-\mathfrak{i} ; T_{j}\right)}{z(z-\mathfrak{i})} e^{-\mathfrak{i} z \ln \frac{K}{L_{j}(0)}} d z .
\end{aligned}
$$

For a fixed $j, j<n-1$, let now $\ln L_{j}$ be given by (17). As noted at the end of Section 3, we may then obtain an additive approximation $\widetilde{X}_{j}\left(T_{j}\right)$ of $X_{j}\left(T_{j}\right)$ via (17) by replacing $\nu^{(j+1)}$ with the approximative compensator

$$
\widetilde{\nu}^{(j+1)}(d t, d u):=\lambda(t) d t p(d u) \prod_{l=j+1}^{n-1} \frac{1+\delta_{l} L_{l}(0) \exp \left(r_{l} u^{\top} \beta_{l}\right)}{1+\delta_{l} L_{l}(0)} .
$$

Hence, approximative caplet prices $\widetilde{C}_{j}(K)$ are obtained from $(27)$, using an approximation $\widetilde{\Phi}_{j+1}$ of the characteristic function $\Phi_{j+1}$, which in turn is obtained by replacing in (11)-(12), $n-1, n$, and $\nu^{(n)}(d t, d u)=\lambda(d t) p(d u)$, respectively with $j, j+1$, and $\widetilde{\nu}^{(j+1)}(d t, d u)$ from (28).

Remark 4 The quality of the approximation in (28) has been analyzed in [14]. In particular they show that this approximation yields good results for typical lengths of tenor structures in practice.

\section{Calibration}

Let us first consider the calibration to a panel of caplets corresponding to maturity $T_{n-1}$ and different strikes $K_{-N}<\cdots<K_{-1}<K_{0}:=L_{n-1}(0)<K_{1}<\cdots<$ $K_{N}$. So, suppose that caplet prices $C_{n-1, j}$ corresponding to $K_{j},-N \leq j \leq N$, are available. We first transform the observations $C_{n-1, j}$ and strikes $K_{j}$ to

$$
\begin{aligned}
O_{n-1, j} & :=\delta_{n-1}^{-1} B_{n}^{-1}(0) L_{n-1}^{-1}(0) C_{n-1, j}-\left(1-e^{v_{j}}\right)^{+}, \\
v_{j} & :=\ln \frac{K_{j}}{L_{j}(0)}, \quad-N \leq j \leq N .
\end{aligned}
$$

Our calibration procedure relies essentially upon the next formula which follows 
from (11), (12), (26), and taking the assumptions of Section 3.5 into account.

$$
\begin{array}{r}
\psi_{n}\left(z ; T_{n-1}\right)=\operatorname{Ln}\left(\Phi_{n}\left(z ; T_{n-1}\right)\right)=\operatorname{Ln}\left(1-z(z+\mathfrak{i}) \mathfrak{F}\left\{\mathcal{O}_{n-1}\right\}(z+\mathfrak{i})\right) \\
=-\frac{\theta_{n-1}^{2} z^{2}}{2}-\mathfrak{i} \varkappa_{n-1} z-\zeta_{n-1}+\zeta_{n-1} \mathfrak{F}\left\{\mu_{n-1}\right\}(z),
\end{array}
$$

with abbreviations

$$
\begin{aligned}
\theta_{n-1}^{2} & :=\left(1-r^{2}\right) \int_{0}^{T_{n-1}}\left|\gamma_{n-1}(s)\right|^{2} d s, \\
\varkappa_{n-1} & :=\lambda T_{n-1} \int_{\mathbb{R}}\left(\exp \left(r u \beta_{n-1, m}(s)\right)-1\right) \mathfrak{p}_{m}(u) d u \\
& +\frac{1}{2} \int_{0}^{T_{n-1}}\left(1-r^{2}\right)\left|\gamma_{n-1}(s)\right|^{2} d s \\
\zeta_{n-1} & :=\lambda T_{n-1}, \\
\mu_{n-1}(\cdot) & :=T_{n-1}^{-1} \int_{0}^{T_{n-1}} r^{-1} \beta_{n-1, m}^{-1}(s) \mathfrak{p}_{m}\left(r^{-1} \beta_{n-1, m}^{-1}(s) \cdot\right) d s,
\end{aligned}
$$

with $\operatorname{Ln}(w):=\ln |w|+\mathfrak{i} \operatorname{Arg} w,-\pi<\operatorname{Arg} w \leq \pi$ denoting the main branch of the logarithm, and $\mathfrak{p}_{m}$ being the density of $p_{m}$ which we now assume to exist.

In principle, the constants $\theta_{n-1}^{2}, \varkappa_{n-1}, \zeta_{n-1}$, and the mixed density $\mu_{n-1}$ can be recovered via (31) from complete knowledge of function $\mathcal{O}_{n-1}$, hence a complete system of model consistent caplet prices $C_{n-1}(K), 0<K<\infty$. Indeed, since $\mathfrak{F}\left\{\mu_{n-1}\right\}(z)$ tends to zero as $|z| \rightarrow \infty$ due to the Riemann-Lebesgue lemma, we have

$$
\begin{aligned}
\theta_{n-1}^{2} & =-2 \lim _{z \rightarrow+\infty} z^{-2} \psi_{n}\left(z ; T_{n-1}\right) \\
\varkappa_{n-1} & =-\lim _{z \rightarrow+\infty} z^{-1} \operatorname{Im} \psi_{n}\left(z ; T_{n-1}\right), \quad \text { and next, } \\
\zeta_{n-1} & =\lim _{z \rightarrow+\infty}\left(-\psi_{n}\left(z ; T_{n-1}\right)-\frac{\theta_{n-1}^{2} z^{2}}{2}-\mathfrak{i} \varkappa_{n-1} z\right),
\end{aligned}
$$

and then the function $\mathfrak{F}\left\{\mu_{n-1}\right\}(z)$ can be found from (31). In practice this approach breaks down due to incomplete knowledge of $\mathcal{O}_{n-1}$ and lack of numerical stability however.

In Belomestny and Reiss (2004) a more stable procedure is developed which estimates all spot characteristics $\theta_{n-1}^{2}, \varkappa_{n-1}, \zeta_{n-1}$, and $\mu_{n-1}(\cdot)$, for a given set of noisy observations (29) due to a discrete set of strikes (30). This procedure consists basically of four steps: (i) first, a continuous piece-wise linear approximation $\widetilde{\mathcal{O}}_{n-1}$ of $\mathcal{O}_{n-1}$ is built from the data; (ii) from $\widetilde{\mathcal{O}}_{n-1}$ an approximation $\widetilde{\psi}_{n}$ of $\psi_{n}$ is obtained; (iii) next the coefficients of the quadratic polynomial on the right-hand side in $(31)$ are estimated from $\widetilde{\psi}_{n}$, under the presence of the nonparametric nuisance part $\mathfrak{F}\left\{\mu_{n-1}\right\}$ (which vanishes at infinity) using appropriate weighting schemes; (iv) finally an estimator for $\mu_{n-1}$ is obtained via FFT inversion of the remainder. The steps (i)-(iv) are spelled out in detail below. 
(i) In view of Appendix 7.4, we construct a continuous piece-wise linear function $v \rightarrow \widetilde{\mathcal{O}}_{n-1}(v)$ on a grid $v_{j},-N-1 \leq j \leq N+1$, with $v_{-N-1} \ll$ $v_{-N}<\cdots<v_{-1}<v_{0}:=0<v_{1}<\cdots<v_{N} \ll v_{N+1}$, , such that $\widetilde{\mathcal{O}}_{n-1}(v)$ fits the data at $v_{j}, j \neq 0, \widetilde{\mathcal{O}}_{n-1}\left(v_{-N-1}\right):=\widetilde{\mathcal{O}}_{n-1}\left(v_{N+1}\right):=0$, and $\widetilde{\mathcal{O}}_{n-1}^{\prime}(0-)-\widetilde{\mathcal{O}}_{n-1}^{\prime}(0+)=1$. The boundary strikes $v_{-N-1}, v_{N+1}$ are included to reflect the fact that $\lim _{v \rightarrow \pm \infty} \mathcal{O}_{n-1}(v)=0$.

(ii) By straightforward FFT we compute $\mathfrak{F}\left\{\widetilde{\mathcal{O}}_{n-1}\right\}(z+\mathfrak{i})$ and so obtain

$$
\widetilde{\psi}_{n}(z):=\operatorname{Ln}\left(1-z(z+\mathfrak{i}) \mathfrak{F}\left\{\widetilde{\mathcal{O}}_{n-1}\right\}(z+\mathfrak{i})\right), \quad z \in \mathbb{R}
$$

(iii) With an estimate $\widetilde{\psi}_{n}$ of $\psi_{n}$ at hand, we obtain estimators for the parametric part $\left(\theta_{n-1}^{2}, \varkappa_{n-1}, \zeta_{n-1}\right)$ by an averaging procedure using the polynomial structure in (31) and the decay property of $\mathfrak{F}\left\{\mu_{n-1}\right\}$. For suitable weight functions $w_{\theta}, w_{\varkappa}$, and $w_{\zeta}$ constructed in Section 5.1, which have bounded support $\mathcal{U}:=[-U, U]$ with $U>0$, and satisfy

$$
\begin{aligned}
& \int w_{\theta} d u=0, \int u^{2} w_{\theta}(u) d u=-2, \quad \int u w_{\varkappa}(u) d u=1 \\
& \int u^{2} w_{\zeta}(u) d u=0, \int w_{\zeta}(u) d u=-1
\end{aligned}
$$

we compute the estimates

$$
\begin{aligned}
\widetilde{\theta}_{n-1}^{2} & :=\int \operatorname{Re}\left(\widetilde{\psi}_{n}(u)\right) w_{\theta}(u) d u, \\
\widetilde{\varkappa}_{n-1} & :=\int \operatorname{Im}\left(\widetilde{\psi}_{n}(u)\right) w_{\varkappa}(u) d u, \\
\widetilde{\zeta}_{n-1} & :=\int \operatorname{Re}\left(\widetilde{\psi}_{n}(u)\right) w_{\zeta}(u) d u,
\end{aligned}
$$

for the parameters $\theta_{n-1}^{2}, \varkappa_{n-1}$, and $\zeta_{n-1}$, respectively.

(iv) The estimate for $\mu_{n-1}$ is obtained via the inverse Fourier transform,

$$
\widetilde{\mu}_{n-1}:=\widetilde{\zeta}_{n-1}^{-1} \mathfrak{F}^{-1}\left\{\left(\widetilde{\psi}_{n}(\cdot)+\frac{\widetilde{\theta}_{n-1}^{2}}{2}(\cdot)^{2}-\mathfrak{i} \widetilde{\varkappa}_{n-1}(\cdot)+\widetilde{\zeta}_{n-1}\right) \mathbf{1}_{\mathcal{U}}\right\}
$$

where $u \in \mathbb{R}$ and $\mathbf{1}_{\mathcal{U}}$ is the indicator function of the set $\mathcal{U}$.

The computational complexity of this estimation procedure is very low. The only time consuming steps are the three integrations in step (iii) and the inverse Fourier transform (inverse FFT) in step (iv). 


\subsection{Determination of the weights $w_{\theta}, w_{\varkappa}$, and $w_{\zeta}$}

Let us assume that for some natural number $p$ and $C>0$,

$$
\max _{0 \leq q \leq p}\left\|\mu_{n-1}^{(q)}\right\|_{L^{2}(\mathbb{R})} \leq C
$$

and consider for some $U>0$ the following weight functions,

$$
\begin{aligned}
w_{\theta}^{U, p}(u) & :=\frac{p+3}{\left(1-2^{-2 /(p+1)}\right) U^{p+3}}|u|^{p}\left(1_{|u / U| \leq 1}-2 \cdot 1_{2^{-1 /(p+1)} \leq|u / U| \leq 1}\right), \\
w_{\varkappa}^{U, p}(u) & :=\frac{p+2}{2 U^{p+2}}|u|^{p} \operatorname{sign}(u) 1_{|u / U| \leq 1}, \\
w_{\zeta}^{U, p}(u) & :=\frac{p+1}{2\left(2^{2 /(p+3)}-1\right) U^{p+1}}|u|^{p}\left(2 \cdot 1_{2^{-1 /(p+3)} \leq|u / U| \leq 1}-1_{|u / U| \leq 1}\right),
\end{aligned}
$$

which satisfy the conditions (36) by straightforwardly checking.

Following Belomestny and Reiss (2005), we can estimate

$$
\begin{aligned}
\left|\widetilde{\theta}_{n-1}^{2}-\theta_{n-1}^{2}\right| \leq & \left|\int \operatorname{Re}\left(\widetilde{\psi}_{n}(u)-\psi_{n}(u)\right) w_{\theta}^{U, p}(u) d u\right| \\
& +\left|\int \operatorname{Re}\left(\mathfrak{F}\left\{\mu_{n-1}\right\}(u)\right) w_{\theta}^{U, p}(u) d u\right|=(1)+(2) .
\end{aligned}
$$

The second term can be estimated using the identity $(\mathfrak{i} u)^{p} \mathfrak{F}\left\{\mu_{n-1}\right\}(u)=$ $\mathfrak{F}\left\{\mu_{n-1}^{(p)}\right\}(u)$, two times Parseval's isometry, and (40),

$$
\begin{aligned}
& \leq\left|\int \mathfrak{F}\left\{\mu_{n-1}\right\}(u) w_{\theta}^{U, p}(u) d u\right|=\left|\int(\mathfrak{i} u)^{p} \mathfrak{F}\left\{\mu_{n-1}\right\}(u) \overline{\left(\frac{w_{\theta}^{U, p}(u)}{\overline{(\mathfrak{i} u)^{p}}}\right)} d u\right| \overline{\left(\frac{w_{\theta}^{U, p}(u)}{\overline{(\mathfrak{i} u)^{p}}}\right)} d u\left|=\frac{1}{2 \pi}\right| \int \mu_{n-1}^{(p)}(s) \mathfrak{F}^{-1}\left\{\frac{w_{\theta}^{U, p}(\cdot)}{\overline{(\mathfrak{i} \cdot)^{p}}}\right\}(s) d s \mid \\
& \leq\left|\int \mathfrak{F}\left\{\mu_{n-1}^{(p)}\right\}(u)\right| \\
& \leq \frac{C}{\sqrt{2 \pi}}\left\|\frac{w_{\theta}^{U, p}(\cdot)}{(\cdot)^{p}}\right\|_{L^{2}(\mathbb{R})}=\frac{C(p+3)}{\sqrt{\pi}\left(1-2^{-2 /(p+1)}\right) U^{p+5 / 2}} \leq C_{1} \frac{(p+1)(p+3)}{U^{p+5 / 2}}
\end{aligned}
$$

for some $C_{1}>0$, which explains the construction of $w_{\theta}^{U, p}$ : for fixed $p$ and $U$ large, (2) falls with $O\left(U^{-(p+5 / 2)}\right)$. The term (1) is due to the noise and lack of data. It can be estimated by

$$
\begin{aligned}
(1) & \leq\left\|\widetilde{\psi}_{n}-\psi_{n}\right\|_{L^{\infty}(\mathcal{U})}\left\|w_{\theta}^{U, p}\right\|_{L^{1}(\mathcal{U})} \\
& =\left\|\widetilde{\psi}_{n}-\psi_{n}\right\|_{L^{\infty}(\mathcal{U})} \frac{2(p+3)}{(p+1)\left(1-2^{-2 /(p+1)}\right) U^{2}} \\
& \leq C_{2}\left\|\widetilde{\psi}_{n}-\psi_{n}\right\|_{L^{\infty}(\mathcal{U})} \frac{p+3}{U^{2}}
\end{aligned}
$$


for some $C_{2}>0$. So we have,

$$
\left|\widetilde{\theta}_{n-1}^{2}-\theta_{n-1}^{2}\right| \leq C_{2}\left\|\widetilde{\psi}_{n}-\psi_{n}\right\|_{L^{\infty}(\mathcal{U})} \frac{p+3}{U^{2}}+C_{1} \frac{(p+1)(p+3)}{U^{p+5 / 2}} .
$$

In a similar way we obtain for $\varkappa_{n-1}$, and $\zeta_{n-1}$,

$$
\begin{aligned}
\left|\widetilde{\varkappa}_{n-1}-\varkappa_{n-1}\right| & \leq C_{3}|| \widetilde{\psi}_{n}-\psi_{n} \|_{L^{\infty}(\mathcal{U})} \frac{p+2}{U(p+1)}+C_{4} \frac{(p+2)}{U^{p+3 / 2}}, \\
\left|\widetilde{\zeta}_{n-1}-\zeta_{n-1}\right| & \leq C_{5}|| \widetilde{\psi}_{n}-\psi_{n} \|_{L^{\infty}(\mathcal{U})}(p+3)+C_{6} \frac{(p+1)(p+3)}{U^{p+1 / 2}},
\end{aligned}
$$

for some $C_{3}, C_{4}, C_{5}, C_{6}>0$. Note that even when $\left\|\mu_{n-1}^{(q)}\right\|_{L_{2}(\mathbb{R})}$ is finite for very large $q$ it is not wise in view of (44) to take $p$ too large. In practice one needs to accomplish that $\left\|\widetilde{\psi}_{n}-\psi_{n}\right\|_{L^{\infty}(\mathcal{U})}$ is small for a large enough $U$ and then $p=1$ or 2 turns out to be a proper choice.

Discussion The order of the error $\left\|\widetilde{\psi}_{n}-\psi_{n}\right\|_{L^{\infty}(\mathcal{U})}$ depends on $N$, the number of caplets available, as well as on the type of errors in caplet prices $C_{n-1, j}$, $-N \leq j \leq N$. For example, assuming that the observed caplet prices are "exact", one can show (see [1]) that

$$
\left\|\widetilde{\psi}_{n}-\psi_{n}\right\|_{L^{\infty}(\mathcal{U})} \leq C_{1} \Delta^{2} U^{2} e^{C_{2} \theta_{n-1}^{2} U^{2}}
$$

with $\Delta=\max _{j=-N, \ldots, N-1}\left(v_{j+1}-v_{j}\right)$ and some positive constants $C_{1}$ and $C_{2}$, provided that $\exp \left(\max \left\{v_{-N},-v_{N}\right\}\right)<\Delta$. So, if $\theta_{n-1}>0$ the convergence rates for $\widetilde{\theta}_{n-1}, \widetilde{\varkappa}_{n-1}$ and $\widetilde{\zeta}_{n-1}$ are logarithmic in $\Delta$ and so correspond to a severely ill-posed problem. The reason for the severe ill-posedness is that we face an underlying deconvolution problem with a Gaussian like distribution: the law of the continuous part of $X_{n-1}$ is convolved with that of the jump part to give the density of $X_{n-1}$.

\section{Correction of $\widetilde{\mu}_{n-1}$}

Due to numerical as well as statistical errors the estimated $\widetilde{\mu}_{n-1}$ may not be a probability density and thus needs to be corrected. Besides that we also want the variance of $X_{n-1}$ to be equal to the Black variance $T_{n-1}\left(\gamma_{n-1}^{B}\right)^{2}$, where

$$
\gamma_{n-1}^{B}:=\sqrt{\frac{1}{T_{n-1}} \int_{0}^{T_{n-1}}\left|\gamma_{n-1}\right|^{2}(s) d s} .
$$

In order to accomplish these requirements we construct a new estimate $\widetilde{\mu}_{n-1}^{+}$as a solution of the following optimization problem,

$$
\left\|\widetilde{\mu}_{n-1}^{+}-\widetilde{\mu}_{n-1}\right\|_{L^{2}(\mathbb{R})}^{2} \rightarrow \min , \quad \inf _{x \in \mathbb{R}} \widetilde{\mu}_{n-1}^{+}(x) \geq 0
$$


subjected to

$$
\int \widetilde{\mu}_{n-1}^{+}(v) d v=1, \quad \int v^{2} \widetilde{\mu}_{n-1}^{+}(v) d v=\frac{T_{n-1}\left(\gamma_{n-1}^{B}\right)^{2}-\widetilde{\theta}_{n-1}^{2}}{\widetilde{\zeta}_{n-1}} .
$$

The solution has a rather simple form and is given by

$$
\widetilde{\mu}_{n-1}^{+}(x ; \xi, \eta):=\max \left\{0, \widetilde{\mu}_{n-1}(x)-\xi-\eta x^{2}\right\}, \quad x \in \mathbb{R},
$$

where $\xi$ and $\eta$ need to be determined such that (46) is satisfied. We note that by representing $\widetilde{\mu}^{+}$as a mixture of given densities, (45)-(46) boils down to a finite dimensional quadratic optimization problem.

Remark 5 The corrected Lévy density $\widetilde{\mu}_{n-1}^{+}(x)$ leads to a similar calibration fit as compared to the uncorrected one. This is illustrated in Figure 1, where the original as well as fitted caplet volas together with estimates for the Lévy density $\mu_{n-1}$ are shown. While in the top row of Figure 1 the results are obtained using the corrected version of $\widetilde{\mu}_{n-1}$, in the bottom row $\widetilde{\mu}_{n-1}$ is employed to compute caplet prices.

\subsection{Procedure for calibration against terminal caplets}

For $U>0$ we denote the estimates (37) obtained using the weight functions (40) by $\theta_{n-1}(U), \varkappa_{n-1}(U), \zeta_{n-1}(U)$, and the corrected Lévy density is denoted by $\widetilde{\mu}_{n-1}^{+}(\cdot ; U)$. From (32) and (33) we can directly infer estimates $r(U)$ and $\lambda(U)$, respectively. We further have to identify a jump density $\mathfrak{p}_{m}$ from $\widetilde{\mu}_{n-1}^{+}(\cdot ; U)$ via (34), while taking into account (25).

Let $\kappa_{\mu^{+}}$and $\alpha_{\mu^{+}}$denote the expectation and the variance, respectively, of a random variable with density $\widetilde{\mu}_{n-1}^{+}(\cdot ; U)$. By plugging $(25)$ into $(34)$, we obtain

$$
\begin{aligned}
\int v \widetilde{\mu}_{n-1}^{+}(v ; U) d v & =\frac{r(U) \kappa_{m}}{\sqrt{1+\lambda(U) \kappa_{m}^{2}}} \frac{1}{T_{n-1}} \int_{0}^{T_{n-1}} \gamma_{n-1, m}(s) d s \\
& =: \frac{r(U) \kappa_{m}}{\sqrt{1+\lambda(U) \kappa_{m}^{2}}} \bar{\gamma}_{n-1, m}=\kappa_{\mu^{+}},
\end{aligned}
$$

from which $\kappa_{m}$ can be solved in principle, and then due to the very construction,

$$
-\mathfrak{F}\left\{\widetilde{\mu}_{n-1}^{+}(\cdot, U)\right\}^{\prime \prime}(0)=\int v^{2} \widetilde{\mu}_{n-1}^{+}(v ; U) d v=\alpha_{\mu^{+}}+\kappa_{\mu^{+}}^{2}=\frac{r^{2}(U)\left(\gamma_{n-1}^{B}\right)^{2}}{\lambda(U)},
$$

which gives combined with (25) and (34), $\int u^{2} \widehat{\mathfrak{p}}_{m}(u ; U) d u=\lambda^{-1}(U)+\kappa_{m}^{2}$. In general, when $\gamma_{n-1, m}$ is time dependent, $\beta_{n-1, m}$ by (25) is time dependent too and so $\mathfrak{p}_{m}$ has to be solved from (34) by a numerical procedure under the above moment restrictions. For example, one could take as an ansatz a mixture of normal densities. However, let us here follow a more simple and pragmatic way and assume that the terminal volatility function is time independent,

$$
\gamma_{n-1, m} \equiv \bar{\gamma}_{n-1, m}=\gamma_{n-1}^{B} .
$$


In this case we obtain from (25) and (34) for the jump density,

$$
\widehat{\mathfrak{p}}_{m}(u ; U):=\frac{r(U) \gamma_{n-1}^{B}}{\sqrt{1+\lambda(U) \kappa_{m}^{2}}} \widetilde{\mu}_{n-1}^{+}\left(\frac{r(U) \gamma_{n-1}^{B}}{\sqrt{1+\lambda(U) \kappa_{m}^{2}}} u ; U\right),
$$

and then combining (47), (48), and (49) yields

$$
\kappa_{m}(U):=\frac{\kappa_{\mu^{+}}}{\sqrt{\lambda(U) \alpha_{\mu^{+}}}} .
$$

Next, substituting (51) in (50) gives

$$
\widehat{\mathfrak{p}}_{m}(u ; U)=\frac{r(U) \gamma_{n-1}^{B}}{\sqrt{1+\kappa_{\mu^{+}}^{2} / \alpha_{\mu^{+}}}} \widetilde{\mu}_{n-1}^{+}\left(\frac{r(U) \gamma_{n-1}^{B}}{\sqrt{1+\kappa_{\mu^{+}}^{2} / \alpha_{\mu^{+}}}} u ; U\right) .
$$

Finally we consider in view of (34),

$$
\begin{aligned}
\widehat{\mu}_{n-1}^{+}(\cdot ; U):= & \frac{1}{T_{n-1}} \int_{0}^{T_{n-1}} \frac{\sqrt{1+\kappa_{\mu^{+}}^{2} / \alpha_{\mu^{+}}}}{r(U) \gamma_{n-1, m}(s)} \times \\
& \times \widehat{\mathfrak{p}}_{m}\left(\frac{\sqrt{1+\kappa_{\mu^{+}}^{2} / \alpha_{\mu^{+}}}}{r(U) \gamma_{n-1, m}(s)} \cdot ; U\right) d s
\end{aligned}
$$

as estimation for $\mu_{n-1}$ used in the log-characteristic function $\widetilde{\psi}_{n}$ for computing terminal caplet model prices.

\section{Choice of $U$}

Based on the results of Section 5.1 we could chose $U$ such that the overall asymptotic error in (42), (43) and (44) is minimized. Putting aside the fact that convergence rates in Section 5.1 are related to the performance of the procedure in the worst situation (the worst $\mu_{n-1}$ satisfying (39)) which is not necessarily the case for the given model, such choice of $U$ depends on $p$, is asymptotic and likely to be inefficient for small sample sizes. As an alternative one can use a data-driven method to choose $U$ (see e.g. Belomestny and Spokoiny (2007)). In the calibration context, however, it seems to be more natural to find $U$ by minimizing the overall calibration error as suggested in Cont and Tankov (2004). The authors in Cont and Tankov (2004) proposed to penalize it with the minimal entropy in order to regularize the underlying optimization problem. In our case the regularization takes place due to the constraints on the functional space we are searching in. While in Cont and Tankov (2004) the minimization is performed over the whole space of Lévy measures (approximated by point masses), we optimize over a specific parametric family (parameterized with $U$ ) of Lévy triplets. We so determine $U$ simply as the solution of the following 
minimization problem

$$
U^{*}=\operatorname{arginf}_{U} \sum_{i=-N}^{N}\left|\widehat{C}_{n-1}\left(K_{i} ; U\right)-C_{n-1, i}\right|^{2},
$$

where $\widehat{C}_{n-1}(\cdot ; U)$ are prices computed from the model due to $\theta_{n-1}(U), \varkappa_{n-1}(U)$, $\zeta_{n-1}(U)$, and $\widehat{\mu}_{n-1}^{+}(\cdot ; U)$.

\subsection{Calibration to other caplets}

With $U^{*}$ is determined via (54) and $\mathfrak{p}_{m}:=\mathfrak{p}_{m}\left(U^{*}\right)$, we introduce the shifted densities

$$
\mathfrak{p}_{j}(u):=\mathfrak{p}_{m}\left(u-\kappa_{j}+\kappa_{m}\right),
$$

hence

$$
\kappa_{j}=\int_{\mathbb{R}} u \widehat{\mathfrak{p}}_{j}(u) d u, \quad j=1, \ldots, m .
$$

Let $\mathcal{U}$ be the upper triangular $m \times m$ matrix with positive diagonal elements such that $\Sigma=\mathcal{U U}^{\top}$. This decomposition exists because $\Sigma$ is invertible. From (21) we then have $A=\lambda^{-1 / 2} \mathcal{U}^{-\top}$. Let us define $\Sigma_{r r^{\prime}}^{(k)}, k \leq r, r^{\prime} \leq m, k=1, \ldots, m$. Since $\mathcal{U}$ is an upper triangular we have $\Sigma^{(k)}=\mathcal{U}^{(k)}\left(\mathcal{U}^{(k)}\right)^{\top}$ and $A^{(k)}=\lambda^{-1 / 2}\left(\mathcal{U}^{(k)}\right)^{-\top}$ with $A^{(k)}$ and $\mathcal{U}^{(k)}$ defined analogously to $\Sigma^{(k)}$. Thus, for knowing $A^{(k)}$ it is sufficient to know $\Sigma^{(k)}$.

We now assume $d=m=n-1$. We determine $\kappa_{j}, j=1, \ldots, n-1$, recursively in the following way. For $j=n-1, \kappa_{n-1}$ is determined from (51), then $\beta_{n-1, n-1}$ from (25), and $\Sigma_{n-1, n-1}^{(n-1)}=\alpha+\kappa_{n-1}^{2}$. Suppose $\beta_{l, k}$ is determined for $l=j, \ldots, n-$ $1, k=l, \ldots, n-1$, where $j>1$. For $j=m=n-1$ we are in the situation of Section 5.2. We then consider the matrix

$$
\Sigma^{(j-1)}\left(\kappa_{j-1}\right):=\left[\begin{array}{l|c}
\alpha+\kappa_{j-1}^{2} & \kappa_{j-1} a^{\top} \\
\kappa_{j-1} a & \Sigma^{(j)}
\end{array}\right]
$$

with $a:=\left[\kappa_{j}, \cdots, \kappa_{n-1}\right]^{\top}$, and where the $(n-j) \times(n-j)$ matrix $\Sigma_{r r^{\prime}}^{(j)}$ is assumed to be already determined. Note that $\alpha=\lambda^{-1}\left(U^{*}\right)$ is the common jump variance. In fact the only unknown parameter to be determined in (56) is $\kappa_{j-1}$. Further, it easily follows that,

$$
\mathcal{U}^{(j-1)}\left(\kappa_{j-1}\right)=\left[\begin{array}{l|l}
\left(\alpha+\kappa_{j-1}^{2}\left(1-a^{\top}\left(\Sigma^{(j)}\right)^{-1} a\right)\right)^{1 / 2} & \begin{array}{c}
\kappa_{j-1} a^{\top}\left(\mathcal{U}^{(j)}\right)^{-\top} \\
\mathcal{U}^{(j)}
\end{array}
\end{array}\right]
$$

and so

$$
\begin{array}{r}
F^{(j-1)}\left(\kappa_{j-1}\right):=\left(\mathcal{U}^{(j-1)}\right)^{-\top}\left(\kappa_{j-1}\right)= \\
{\left[\begin{array}{c|c}
\left(\alpha+\kappa_{j-1}^{2}\left(1-a^{\top}\left(\Sigma^{(j)}\right)^{-1} a\right)\right)^{-1 / 2} & \\
-\left(\alpha+\kappa_{j-1}^{2}\left(1-a^{\top}\left(\Sigma^{(j)}\right)^{-1} a\right)\right)^{-1 / 2} \kappa_{j-1} a & \left(\mathcal{U}^{(j)}\right)^{-\top}
\end{array}\right]}
\end{array}
$$


Next, set according to (20)

$$
\begin{aligned}
& \beta_{j-1, k}\left(\kappa_{j-1}\right)=\lambda^{-1 / 2} \sum_{r=j-1}^{k} F_{k, r}^{(j-1)}\left(\kappa_{j-1}\right) \gamma_{j-1, r}, \quad k=j-1, \ldots, n-1, \\
& \beta_{j-1, k}\left(\kappa_{j-1}\right)=0, \quad 1 \leq k<j-1 .
\end{aligned}
$$

By a simple trial and error search we then determine $\kappa_{j-1}$ such that the least squares fit error of the $T_{j-1}$ caplet panel is as small as possible. For each guess of $\kappa_{j-1}$ the model caplet prices may be computed by Monte Carlo simulation of the model, or as an alternative by approximating caplet prices as proposed at the end of Section 4.

\section{Calibration to real data}

In this section we calibrate the model (7) to market data given on 11.01.2004. The caplet-strike volatility matrix is partially shown in Table 1 . The corre-

\begin{tabular}{|c|cccccccc|}
\hline $\mathrm{K} / \mathrm{T}$ & 0.150 & 0.200 & 0.225 & 0.250 & 0.300 & 0.400 & 0.500 & 0.600 \\
\hline \hline 0.50 & 0.2604 & 0.1735 & 0.1819 & 0.1969 & 0.2453 & 0.2708 & 0.3197 & 0.3407 \\
0.75 & 0.2678 & 0.2036 & 0.2052 & 0.2136 & 0.2401 & 0.2598 & 0.3052 & 0.3258 \\
1.75 & 0.2832 & 0.2587 & 0.2475 & 0.2365 & 0.2227 & 0.2246 & 0.2539 & 0.2733 \\
2.50 & 0.2850 & 0.2651 & 0.2513 & 0.2334 & 0.2125 & 0.2051 & 0.2234 & 0.2412 \\
3.50 & 0.2804 & 0.2581 & 0.2432 & 0.2233 & 0.2016 & 0.1856 & 0.1924 & 0.2071 \\
4.50 & 0.2720 & 0.2474 & 0.2319 & 0.2142 & 0.1934 & 0.1720 & 0.1711 & 0.1821 \\
5.50 & 0.2625 & 0.2381 & 0.2219 & 0.2079 & 0.1872 & 0.1625 & 0.1566 & 0.1640 \\
6.50 & 0.2531 & 0.2314 & 0.2144 & 0.2039 & 0.1824 & 0.1557 & 0.1470 & 0.1510 \\
7.50 & 0.2447 & 0.2270 & 0.2092 & 0.2016 & 0.1788 & 0.1510 & 0.1407 & 0.1418 \\
8.50 & 0.2375 & 0.2241 & 0.2058 & 0.2002 & 0.1761 & 0.1477 & 0.1367 & 0.1355 \\
9.50 & 0.2315 & 0.2224 & 0.2036 & 0.1995 & 0.1740 & 0.1454 & 0.1342 & 0.1311 \\
11.50 & 0.2212 & 0.2206 & 0.2011 & 0.1988 & 0.1707 & 0.1424 & 0.1312 & 0.1253 \\
14.50 & 0.2149 & 0.2201 & 0.2003 & 0.1987 & 0.1689 & 0.1410 & 0.1302 & 0.1228 \\
19.50 & 0.2111 & 0.2200 & 0.2001 & 0.1987 & 0.1678 & 0.1404 & 0.1300 & 0.1219 \\
\hline
\end{tabular}

Table 1: Caplet volatilities $\sigma_{T}^{K}$ for different strikes and different tenor dates (in years).

sponding implied volatility surface is shown in Figure 2.

Pronounced smiles are clearly observable. Due to the structure of the given data we are going to calibrate the jump diffusion model based on semi-annual tenors, i.e. $\delta_{j} \equiv 0.5$, with $n=41$, and where the initial calibration date 01.11.04 is identified with $T_{0}=0$. 
In a pre-calibration a standard market model is calibrated to ATM caps and ATM swaptions using Schoenmakers (2005). However, we emphasize that the method by which this input market model is obtained is not essential nor a discussion point for this paper. For the pre-calibration we have used a volatility structure of the form

$$
\gamma_{i}(t)=c_{i} g\left(T_{i}-t\right) e_{i}, \quad 0 \leq t \leq \min \left(T_{i}, T_{j}\right), \quad 1 \leq i, j<n,
$$

where $g$ is a simple parametric function and $e_{i}$ are unit vectors. The calibration routine returned $e_{i} \in \mathbb{R}^{40}$ with

$$
e_{i}^{\top} e_{j}=\rho_{i j}=\exp [-0.005|i-j|] \quad 1 \leq i, j<41,
$$

such that the matrix $\left(e_{i, k}\right)$ is upper triangular, and

$$
g(s)=0.8+0.2 e^{-2.0 s} .
$$

The $c_{i}$ can be readily computed from

$$
\left(\sigma_{T_{i}}^{A T M}\right)^{2} T_{i}=c_{i}^{2} \int_{0}^{T_{i}} g^{2}(s) d s, \quad i=1, \ldots, n-1,
$$

using the initial Libor curve, which is obtained by a standard stripping procedure from the yield curve at 11.01.04, and is given in Table 2 .

\begin{tabular}{|r|r|r|r|r|r|r|r|}
\hline$T_{i}$ & $L_{i}(0)$ & $T_{i}$ & $L_{i}(0)$ & $T_{i}$ & $L_{i}(0)$ & $T_{i}$ & $L_{i}(0)$ \\
\hline \hline 0.5 & 0.0238176 & 5.5 & 0.0451931 & 10.5 & 0.0509249 & 15.5 & 0.0539696 \\
\hline 1 & 0.0264201 & 6 & 0.0465074 & 11 & 0.0512114 & 16 & 0.0540521 \\
\hline 1.5 & 0.0292798 & 6.5 & 0.0475881 & 11.5 & 0.0515804 & 16.5 & 0.0540931 \\
\hline 2 & 0.0320656 & 7 & 0.0484201 & 12 & 0.0520317 & 17 & 0.0540933 \\
\hline 2.5 & 0.0345508 & 7.5 & 0.0490942 & 12.5 & 0.0524639 & 17.5 & 0.054053 \\
\hline 3 & 0.0366693 & 8 & 0.0496402 & 13 & 0.0528456 & 18 & 0.0539728 \\
\hline 3.5 & 0.0385821 & 8.5 & 0.0500331 & 13.5 & 0.0531757 & 18.5 & 0.0538533 \\
\hline 4 & 0.040381 & 9 & 0.0502848 & 14 & 0.0534529 & 19 & 0.053695 \\
\hline 4.5 & 0.0420863 & 9.5 & 0.0504889 & 14.5 & 0.0536757 & 19.5 & 0.0534984 \\
\hline 5 & 0.0437079 & 10 & 0.0506932 & 15 & 0.0538451 & 20 & 0.053268 \\
\hline
\end{tabular}

Table 2: Initial Libor curve.

The further steps are as follows

1. The model for $L_{n-1}$ is calibrated as described in Section 5.2 and the calibrated parameters are shown in Table 3 . The calibrated density $p_{n-1}(x)$ is plotted in Figure 3. Note that the variance of the distribution corresponding to $p_{n-1}$ is equal to $1 / \lambda=10.0$ in order to ensure (24). 


\begin{tabular}{|c|c|c|}
\hline$r$ & $\lambda$ & $\kappa_{m}$ \\
\hline \hline 0.7 & 0.1 & -0.005 \\
\hline
\end{tabular}

Table 3: Parameters calibrated using terminal caplet volas $\sigma_{T_{n-1}}^{K}$.

2. Remaining parameters $\kappa_{j}, j=1, \ldots, 39$, are calibrated sequentially as described in Section 5.3 with approximation formula (28) being used for pricing caplets. It turned out experimentally that $\kappa_{j}$ can be taken on the line

$$
\kappa_{j}=\kappa_{40}-0.0751 *(40-j), \quad j=40, \ldots, 1 .
$$

The quality of the calibration can be seen in Figure 4, where calibrated volatility curves are shown for several caplet maturities together with original caplet volas and ATM caplet volas. The overall root-mean-square fit we have reached shows to be $0.5 \%-5 \%$, when the number of caplet panels ranges from 2 to 20 . Fitting all the 40 caplet panels with an acceptable accuracy (e.g. $\leq 5 \%$ ), would require a more flexible structure for $\mathfrak{p}_{j}$, $j<n-1$, however.

For comparison we also present results for a newer data set, 22.10.2008. Figure 5 shows original and fitted caplet implied volatilities for different maturities as well as estimated densities $\widetilde{\mu}_{n-1}^{+}\left(x, U^{*}\right)$. In the top of Figure 5 the results for 11.01.2004 are presented, whereas the bottom figure corresponds to 22.10.08. As we can see, the implied volatility curves have quite different shapes for these two data sets, nevertheless the jump diffusion Libor model is able to reproduce both of them with an acceptable accuracy. Note that the estimated Lévy measure for 22.10 .08 puts much more mass on negative jumps compared to the Lévy measure calibrated at 11.01.04. This may be an indication that in crisis times prices are more strongly influenced by uncertainty about future and negative expectations.

\section{Appendix}

\subsection{Equivalence of (1) and (3)}

Suppose on $\left(\Omega, \mathcal{F}, \mathcal{F}_{t}, P_{n}\right)$ we are given $\eta$ and $W^{(n)}$ as in $(3)$, and for $k=1, \ldots, m$ we are given a random measure $\mu_{k}$ on $\mathbb{R}_{+} \times E_{k}$, with $E_{k}$ Lusin, of the form (2)

$$
\mu_{k}=\sum_{n \geq 1} 1_{T_{n}^{(k)}(\omega)=t} \delta_{\left(t, \beta_{t}^{(k)}(\omega)\right)}(d t, d u),
$$

where the stopping times $\left(T_{n}^{(k)}\right)_{k=1, \ldots, m, n \geq 1}$ satisfy $T_{n}^{(k)}(\omega) \neq T_{m}^{(l)}(\omega)$ for $n \neq m$ or $k \neq l$. Further let for $i=1, \ldots, n-1, k=1, \ldots, m$, the $E_{k}$-valued function processes $\psi_{i k}$ be predictable. By treating $E_{k}$ and $E_{l}$ for $k \neq l$ as completely 
different spaces, i.e. $E_{k} \cap E_{l}=\varnothing$ (which may be achieved by giving them different colors if need be), we may construct straightforwardly the Lusin space $E:=$ $\bigcup_{k=1}^{m} E_{k}$ and define a random measure $\mu:=\sum_{k=1}^{m} \mu_{k}$ on $\mathbb{R}_{+} \times E$. Let now $\nu_{k}^{(n)}$ be the $\left(P_{n}, \mathcal{F}\right)$-compensator of $\mu_{k}$ (which is concentrated on $E_{k}$ ), then it easily follows that $\nu^{(n)}:=\sum_{k=1}^{m} \nu_{k}^{(n)}$ is the $\left(P_{n}, \mathcal{F}\right)$-compensator of $\mu$, and by defining $\psi_{i}(t, u, \omega):=\psi_{i k}(t, u, \omega)$ if $u \in E_{k},(3)$ may be written as (1).

\subsection{Proof of Lemma 3}

Proof of (i):

$$
\begin{aligned}
E e^{\mathfrak{i} z^{\top} J(t)} & =E E\left[e^{\mathfrak{i} z^{\top}{ }_{l=1}^{N_{t}} \varphi\left(s_{l}, u_{l}\right)} \mid N_{t}\right]=E\left[\prod_{l=1}^{N_{t}} e^{\mathfrak{i} z^{\top} \varphi\left(s_{l}, u_{l}\right)} \mid N_{t}\right] \\
& =E\left(\int_{0}^{t} \frac{\lambda(s) d s}{\int_{0}^{t} \lambda(\tau) d \tau} \int_{E} e^{\mathfrak{i} z^{\top} \varphi(s, u)} p(d u)\right)^{N_{t}} \\
& =\sum_{k=0}^{\infty} \frac{\left(\int_{0}^{t} \lambda(\tau) d \tau\right)^{k}}{k !} e^{-{ }_{0}^{t} \lambda(\tau) d \tau}\left(\int_{0}^{t} \frac{\lambda(s) d s}{\int_{0}^{t} \lambda(\tau) d \tau} \int_{E} e^{\mathfrak{i} z^{\top} \varphi(s, u)} p(d u)\right)^{k} \\
& =\exp \int_{0}^{t} \lambda(s) d s \int_{E}\left(e^{\mathfrak{i} z^{\top} \varphi(s, u)}-1\right) p(d u) .
\end{aligned}
$$

Proof of (ii): By differentiating the characteristic function with respect to $z_{l}$ and $z_{l}^{\prime}$ we obtain

$$
\begin{gathered}
\frac{\partial}{\partial z_{l}} E e^{\mathfrak{i} z^{\top} J(t)}=\mathfrak{i} \int_{0}^{t} \lambda(s) d s \int_{E} e^{\mathfrak{i} z^{\top} \varphi(s, u)} \varphi_{l}(s, u) p(d u) \cdot \\
\cdot \exp \int_{0}^{t} \lambda(s) d s \int_{E}\left(e^{\mathfrak{i} z^{\top} \varphi(s, u)}-1\right) p(d u), \\
\frac{\partial^{2}}{\partial z_{l} \partial z_{l^{\prime}}} E e^{\mathfrak{i} z^{\top} J(t)}=-\int_{0}^{t} \lambda(s) d s \int_{E} e^{\mathfrak{i} z^{\top} \varphi(s, u)} \varphi_{l^{\prime}}(s, u) p(d u) \cdot \\
\int_{0}^{t} \lambda(s) d s \int_{E} e^{\mathfrak{i} z^{\top} \varphi(s, u)} \varphi_{l}(s, u) p(d u) \exp \int_{0}^{t} \lambda(s) d s \int_{E}\left(e^{\mathfrak{i} z^{\top} \varphi(s, u)}-1\right) p(d u) \\
-\int_{0}^{t} \lambda(s) d s \int_{E} e^{\mathfrak{i} z^{\top} \varphi(s, u)} \varphi_{l}(s, u) \varphi_{l^{\prime}}(s, u) p(d u) \\
\cdot \exp \int_{0}^{t} \lambda(s) d s \int_{E}\left(e^{\mathfrak{i} z^{\top} \varphi(s, u)}-1\right) p(d u) .
\end{gathered}
$$

Hence

$$
E J_{l}(t)=\int_{0}^{t} \lambda(s) d s \int_{E} \varphi_{l}(s, u) p(d u)
$$


and

$$
\begin{aligned}
E J_{l}(t) J_{l^{\prime}}(t) & =\int_{0}^{t} \lambda(s) d s \int_{E} \varphi_{l^{\prime}}(s, u) p(d u) \cdot \int_{0}^{t} \lambda(s) d s \int_{E} \varphi_{l}(s, u) p(d u) \\
& +\int_{0}^{t} \lambda(s) d s \int_{E} \varphi_{l}(s, u) \varphi_{l^{\prime}}(s, u) p(d u),
\end{aligned}
$$

and then note that $\operatorname{Cov}\left(J_{l}(t), J_{l^{\prime}}(t)\right)=E J_{l}(t) J_{l^{\prime}}(t)-E J_{l}(t) E J_{l^{\prime}}(t)$.

\subsection{Summed reciprocal eigenvalues of $\Sigma$}

Consider the determinant

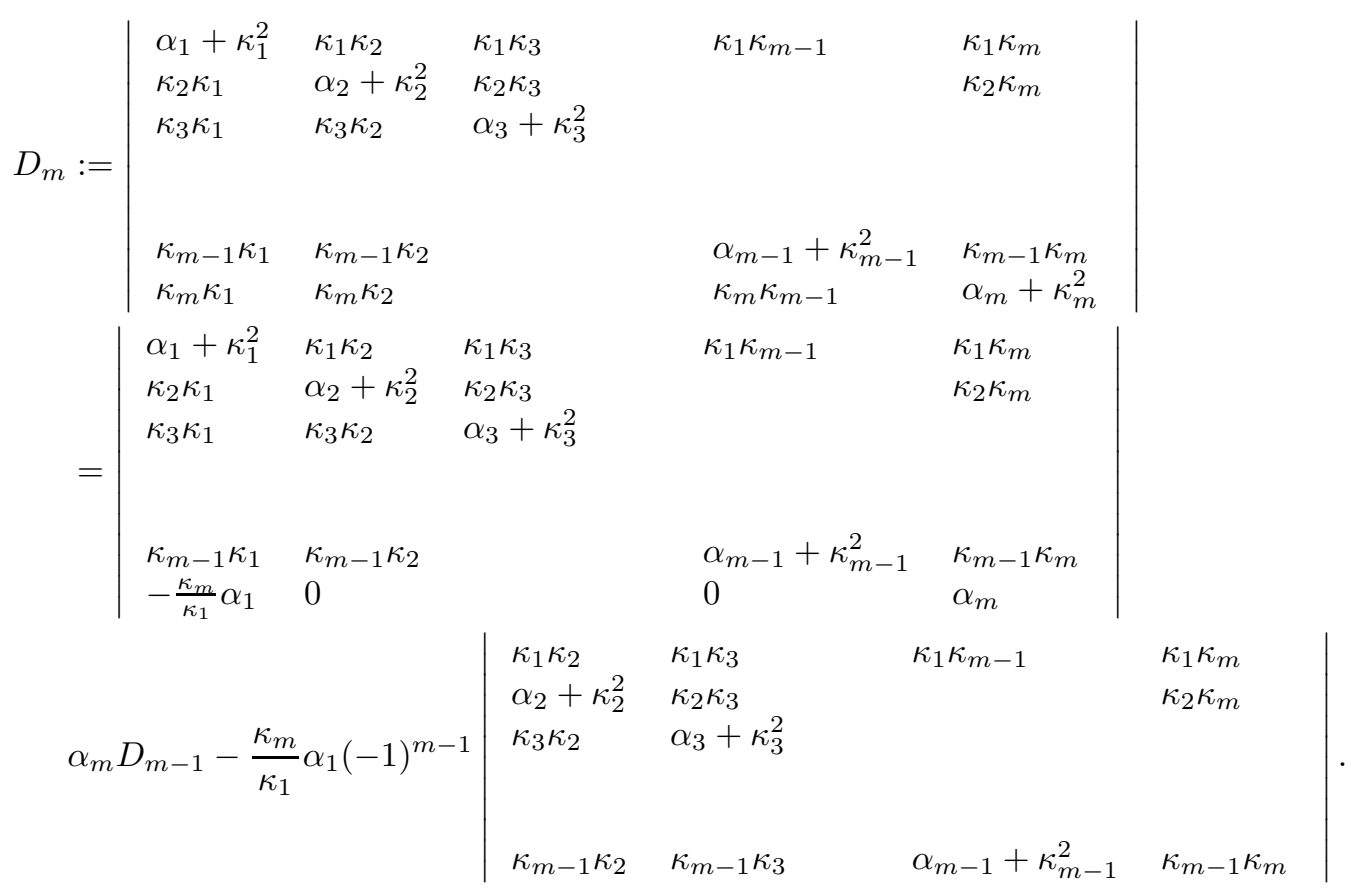

Since

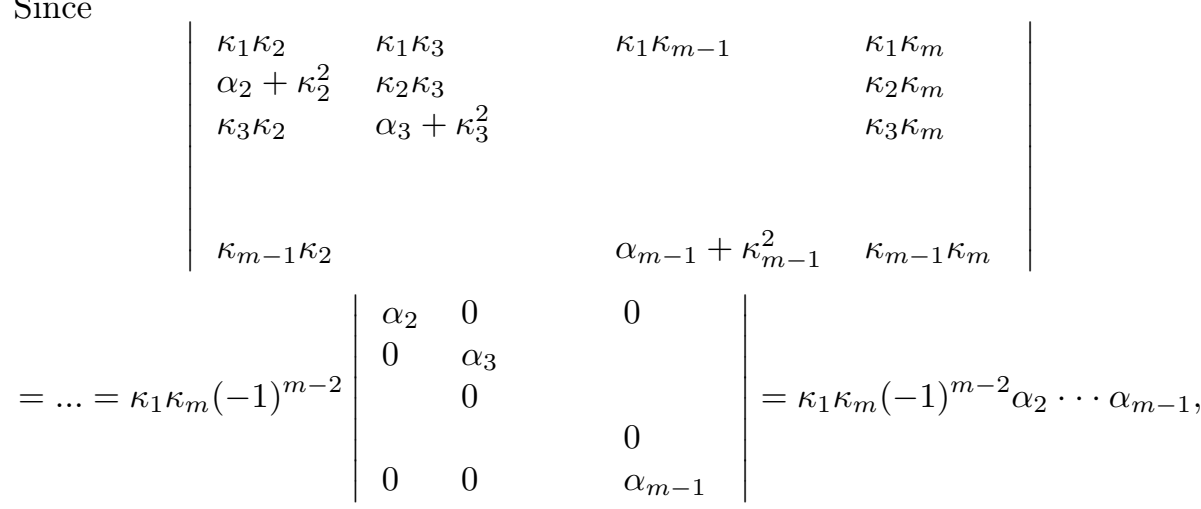


we obtain

$$
\begin{aligned}
D_{m} & =\alpha_{m} D_{m-1}-\frac{\kappa_{m}}{\kappa_{1}} \alpha_{1}(-1)^{m-1} \kappa_{1} \kappa_{m}(-1)^{m-2} \alpha_{2} \cdots \alpha_{m-1} \\
& =\alpha_{m} D_{m-1}+\kappa_{m}^{2} \alpha_{1} \alpha_{2} \cdots \alpha_{m-1}=\ldots \\
& =\left(1+\sum_{p=1}^{m} \frac{\kappa_{p}^{2}}{\alpha_{p}}\right) \prod_{q=1}^{m} \alpha_{p} .
\end{aligned}
$$

Hence,

$$
\begin{aligned}
D_{m}(\lambda) & =\left|\Sigma-\lambda I_{m}\right|=\left(1+\sum_{p=1}^{m} \frac{\kappa_{p}^{2}}{\alpha_{p}-\lambda}\right) \prod_{q=1}^{m}\left(\alpha_{q}-\lambda\right) \\
& =\prod_{q=1}^{m}\left(\alpha_{q}-\lambda\right)+\sum_{p=1}^{m} \kappa_{p}^{2} \prod_{\substack{q=1, q \neq p}}^{m}\left(\alpha_{q}-\lambda\right)=: \cdots+K \lambda+|\Sigma|,
\end{aligned}
$$

where the coefficient of $\lambda$ is given by

$$
K:=-\sum_{p=1}^{m} \prod_{\substack{q=1, q \neq p}}^{m} \alpha_{q}-\sum_{p=1}^{m} \sum_{\substack{r=1, r \neq p}}^{m} \kappa_{p}^{2} \prod_{\substack{q=1, q \neq p, q \neq r}}^{m} \alpha_{q} .
$$

We finally obtain

$$
\begin{aligned}
& \sum_{p=1}^{m} \frac{1}{\lambda_{i}}=-\frac{K}{|\Sigma|}=\frac{\sum_{p=1}^{m} \prod_{\substack{q=1, q \neq p}}^{m} \alpha_{q}+\sum_{\substack{p=1 \\
r=1, r \neq p}}^{m} \kappa_{p}^{2} \prod_{\substack{q=1, q \neq p, q \neq r}}^{m} \alpha_{q}}{\prod_{q=1}^{m} \alpha_{q}+\sum_{p=1}^{m} \kappa_{p}^{2} \prod_{\substack{q=1, q \neq p}}^{m} \alpha_{q}} \\
& \sum_{p=1}^{m} \frac{1}{\alpha_{p}}+\sum_{p=1}^{m} \sum_{r=1}^{m} \frac{\kappa_{p}^{2}}{\alpha_{p} \alpha_{r}} \\
& =\frac{r \neq p}{1+\sum_{p=1}^{m} \frac{\kappa_{p}^{2}}{\alpha_{p}}} .
\end{aligned}
$$

\subsection{Characteristic properties of $\mathcal{O}_{j}$}

By denoting the density of $L_{j}\left(T_{j}\right)$ with $\rho_{L_{j}\left(T_{j}\right)}$ we may write

$$
\begin{aligned}
C_{j}(K) & =B_{j+1}(0) E_{j+1}\left[\left(L_{j}\left(T_{j}\right)-K\right)^{+} \delta_{j}\right] \\
& =B_{j+1}(0) \delta_{j} \int_{K}^{\infty}(y-K) \rho_{L_{j}\left(T_{j}\right)}(y) d y
\end{aligned}
$$


and then by differentiating two times with respect to $K$ we obtain

$$
C_{j}^{\prime \prime}(K)=B_{j+1}(0) \delta_{j} \rho_{L_{j}\left(T_{j}\right)}(K) .
$$

The density of $X_{j}:=\ln L_{j}\left(T_{j}\right)-\ln L_{j}(0)$ is obviously given by $\rho_{X_{j}}(v):=$ $\rho_{L_{j}\left(T_{j}\right)}\left(L_{j}(0) e^{v}\right) L_{j}(0) e^{v}$, so

$$
\begin{aligned}
\rho_{X_{j}}(v) & =B_{j+1}^{-1}(0) \delta_{j}^{-1} C_{j}^{\prime \prime}\left(L_{j}(0) e^{v}\right) L_{j}(0) e^{v} \\
& =B_{j+1}^{-1}(0) \delta_{j}^{-1} L_{j}^{-1}(0)\left(\mathcal{C}_{j}^{\prime \prime}(v)-\mathcal{C}_{j}^{\prime}(v)\right) e^{-v} \\
& =\left(\mathcal{O}_{j}^{\prime \prime}(v)-\mathcal{O}_{j}^{\prime}(v)\right) e^{-v}, v \neq 0,
\end{aligned}
$$

where $\mathcal{O}_{j}^{\prime \prime}-\mathcal{O}_{j}^{\prime}$ extends continuously at $v=0$. In particular, $\mathcal{O}_{j}$ satisfies

$$
\mathcal{O}_{j}^{\prime \prime}(v)-\mathcal{O}_{j}^{\prime}(v)>0 \quad \text { and } \quad \mathcal{O}^{\prime}(0-)-\mathcal{O}^{\prime}(0+)=1 .
$$

On the grid $v_{j},-N-1 \leq j \leq N+1$ we consider a continuous piecewise linear approximation $\widetilde{\mathcal{O}}_{n-1}$ of $\mathcal{O}_{n-1}$,

$$
\begin{gathered}
\widetilde{\mathcal{O}}_{n-1}(v):= \\
\sum_{j=-N}^{N+1} \frac{1}{v_{j}-v_{j-1}}\left(O_{n-1, j-1} v_{j}-v_{j-1} O_{n-1, j}+v\left(O_{n-1, j}-O_{n-1, j-1}\right)\right) 1_{\left[v_{j-1}, v_{j}\right)}(v)
\end{gathered}
$$

with $v_{j}$ and $O_{n-1, j-1}$ given by (29) and (30), extended with $O_{n-1,-N-1}=$ $O_{N+1, n-1}=0$ (note that $v_{0}:=0$ ). Then it follows that (with suppressed subscript $n-1)$

$$
\frac{d}{d v}^{\text {distr }} \widetilde{\mathcal{O}}(v)=\sum_{j=-N}^{N+1} \frac{O_{j}-O_{j-1}}{v_{j}-v_{j-1}} 1_{\left[v_{j-1}, v_{j}\right)}(v)
$$

in (Schwartz) distribution sense. Differentiating in distribution again yields

$$
\begin{aligned}
{\frac{d^{2}}{d v^{2}}}^{\text {distr }} \widetilde{\mathcal{O}}(v) & =\frac{O_{-N}}{v_{-N}-v_{-N-1}} \delta_{v_{-N-1}}+\frac{O_{N}}{v_{N+1}-v_{N}} \delta_{v_{N+1}} \\
& +\sum_{j=-N}^{N}\left(\frac{O_{j+1}-O_{j}}{v_{j+1}-v_{j}}-\frac{O_{j}-O_{j-1}}{v_{j}-v_{j-1}}\right) \delta_{v_{j}} .
\end{aligned}
$$

Because $\mathcal{O}$ satisfies

$$
\mathcal{O}^{\prime \prime}(v)-\mathcal{O}^{\prime}(v)={\frac{d^{2}}{d v^{2}}}^{\text {distr }} \mathcal{O}-\frac{d}{d v}^{\text {distr }} \mathcal{O}, \quad v \neq 0
$$


we consider for $v \neq 0$,

$$
\begin{aligned}
& \left(\frac{d^{2}}{d v^{2}}{ }^{\text {distr }} \widetilde{\mathcal{O}}-\frac{d}{d v}^{\text {distr }} \widetilde{\mathcal{O}}\right) e^{-v}=-\frac{O_{1}-O_{0}}{v_{1}} 1_{\left[0, v_{1}\right)}(v) e^{-v}+\frac{O_{N}}{v_{N+1}-v_{N}} \delta_{v_{N+1}} e^{-v_{N+1}} \\
& \quad \frac{O_{-N}}{v_{-N}-v_{-N-1}} \delta_{v_{-N-1}} e^{-v_{-N-1}}-\frac{O_{-N}}{v_{-N}-v_{-N-1}} 1_{\left[v_{-N-1}, v_{-N}\right)}(v) e^{-v} \\
& +\sum_{\substack{j=-N \\
j \neq 0}}^{N}\left[\left(\frac{O_{j+1}-O_{j}}{v_{j+1}-v_{j}}-\frac{O_{j}-O_{j-1}}{v_{j}-v_{j-1}}\right) \delta_{v_{j}} e^{-v_{j}}-\frac{O_{j+1}-O_{j}}{v_{j+1}-v_{j}} 1_{\left[v_{j}, v_{j+1}\right)}(v) e^{-v}\right]
\end{aligned}
$$

which follows from (58) and (59) and some rearranging of terms. Since the generalised function (60) should be an approximation of the density $\rho_{X_{n-1}}$, integrals over each interval $\left[v_{j-1}, v_{j}\right), j=-N, . . N+1$, should be non-negative. This leads to

$$
\begin{aligned}
0 & \leq\left(\frac{O_{j+1}-O_{j}}{v_{j+1}-v_{j}}-\frac{O_{j}-O_{j-1}}{v_{j}-v_{j-1}}\right) e^{-v_{j}}-\frac{O_{j+1}-O_{j}}{v_{j+1}-v_{j}} \int 1_{\left[v_{j}, v_{j+1}\right)}(v) e^{-v} d v \\
& =\frac{O_{j+1}-O_{j}}{v_{j+1}-v_{j}} e^{-v_{j+1}}-\frac{O_{j}-O_{j-1}}{v_{j}-v_{j-1}} e^{-v_{j}}, \quad j=-N, \ldots,-N, \quad j \neq 0 .
\end{aligned}
$$

Note that (61) holds if the input data are consistent with a function $\mathcal{O}$ which is convex on both $v<0$ and $v>0$, and if the grid $v_{j}$ is fine enough. Further, the total mass of (60) should be one. This leads straightforwardly to the requirement,

$$
\frac{O_{0}-O_{-1}}{-v_{-1}}-\frac{O_{1}-O_{0}}{v_{1}}=1,
$$

which is a discretisation of the boundary condition (57) in fact.

\section{References}

[1] Belomestny, D. and Reiß, M. (2006). Spectral calibration of exponential Lévy models, Finance and Stochastics, 10, 449-474.

[2] Belomestny, D. and Spokoiny, V. (2007). Local-likelihood modeling via stage-wise aggregation. Annals of Statistics, 35(5), 2287-2311.

[3] Belomestny, D. and Schoenmakers, J. (2006). A jump-diffusion Libor model and its robust calibration, Preprint 1113, Weierstraß Institute (WIAS) Berlin.

[4] Brigo, D. and Mercurio, F. (2001). Interest rate models-theory and practice. Springer Finance. Springer-Verlag, Berlin.

[5] Brace, A., Gatarek, D. and Musiela, M. (1997). The Market Model of Interest Rate Dynamics, Mathematical Finance, 7(2), 127-155. 
[6] Carr, P. and Madan, D. (1999). Option Valuation Using the Fast Fourier Transform, Journal of Computational Finance, 2, 61-74.

[7] Cont, R. and Tankov, P. (2003). Financial Modelling with Jump processes, Chapman \& Hall.

[8] Cont, R., and Tankov, P. (2004). Nonparametric calibration of jumpdiffusion option pricing models, Journal of Computational Finance, 7(3), $1-49$.

[9] Eberlein, E. and Keller, U. (1995). Hyperbolic distributions in finance, Bernoulli 1(3), 281-299.

[10] Eberlein, E., Keller, U. and Prause, K. (1998). New insights into smile, mispricing, and value at risk: the hyperbolic model, Journal of Business, 71(3), 371-405.

[11] Eberlein, E. and Özkan, F. (2005). The Lévy LIBOR model, Finance Stoch. 7(1), 1-27.

[12] Glasserman, P. Monte Carlo methods in financial engineering. Applications of Mathematics (New York), 53. Stochastic Modelling and Applied Probability. Springer-Verlag, New York, 2004.

[13] Glasserman, P. and Kou, S. G. (2003). The term structure of simple forward rates with jump risk. Math. Finance 13(3), 383-410.

[14] Glasserman, P. and Merener, N. (2003). Cap and swaption approximations in Libor market models with jumps, Journal of Computational Finance, $7(1), 1-36$.

[15] Jamshidan, F. (1997). LIBOR and swap market models and measures, Finance and Stochastics, 1, 293-330.

[16] Jamshidian, F. (2001). LIBOR Market Model with Semimartingales, in "Option Pricing, Interest Rates and Risk Management", Cambridge Univ.

[17] Kurbanmuradov, O., Sabelfeld, K. and Schoenmakers, J. (2002). Lognormal approximations to Libor market models, Journal of Computational Finance, 6(1), 69-100.

[18] Schoenmakers, J. (2005). Robust Libor Modelling and Pricing of Derivative Products. Boca Raton London New York Singapore, Chapman \& Hall - CRC Press. 

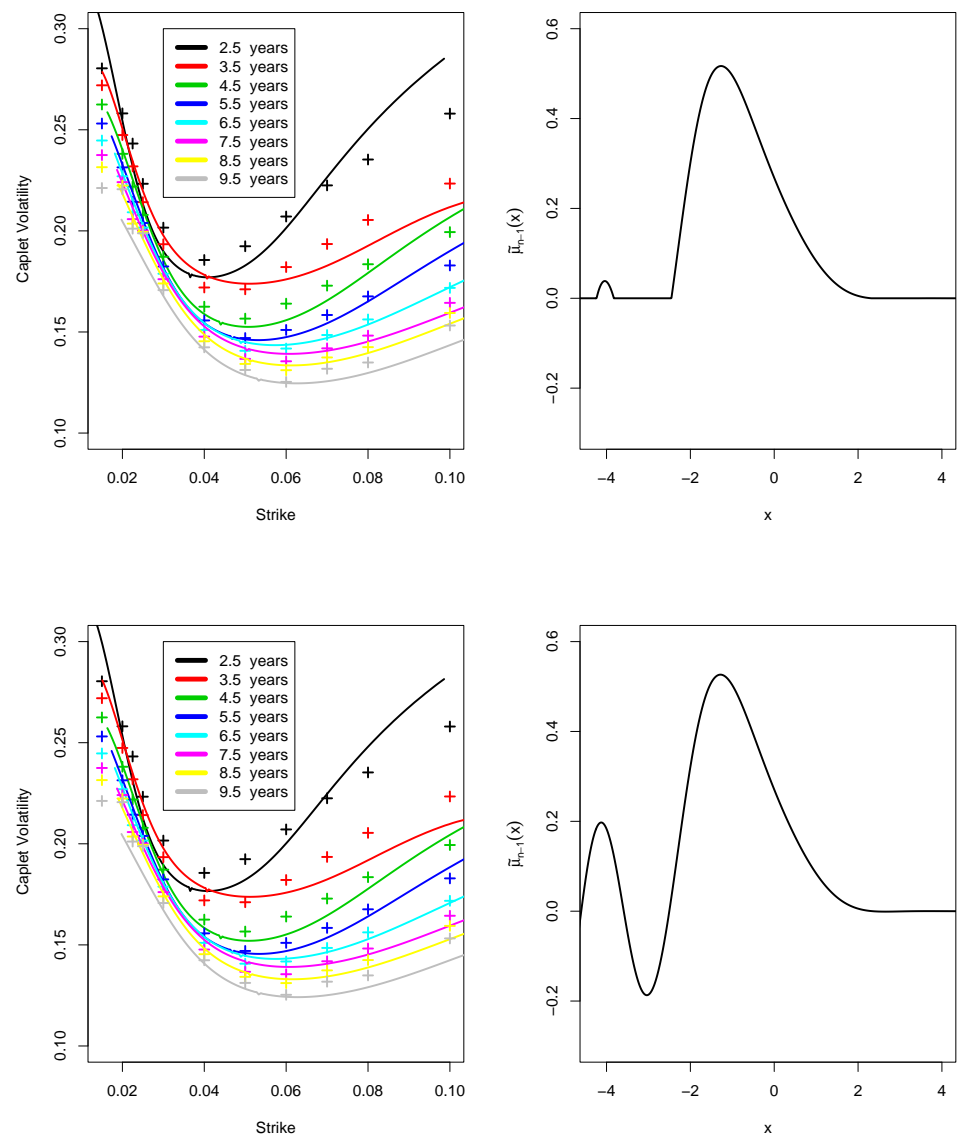

Figure 1: Caplet volas from the calibrated model (solid lines), original caplet volas $\sigma_{T}^{K}$ (crosses) for different caplet maturities $T$ on the left hand side, and corrected (top) and uncorrected(bottom) estimates for Lévy density $\mu_{n-1}$ on the right hand side. 
Caplets Implied Volatilities

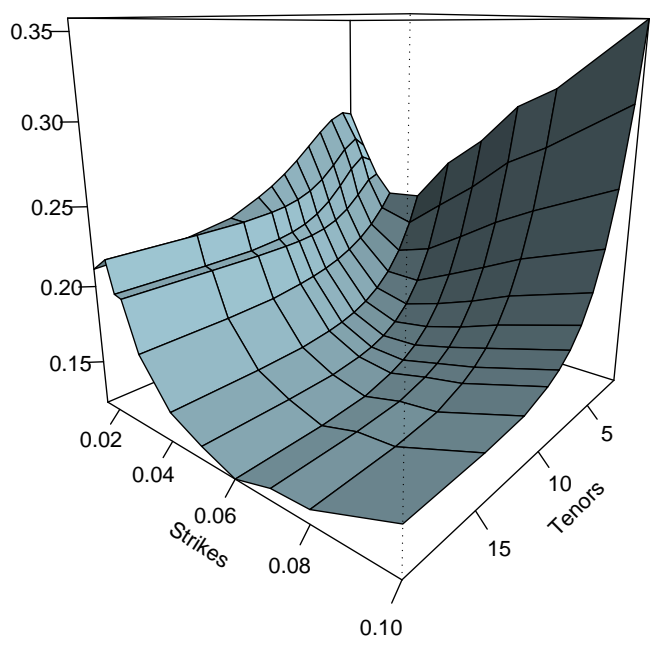

Figure 2: Smoothed caplet implied volatility surface $\sigma_{T}^{K}$.

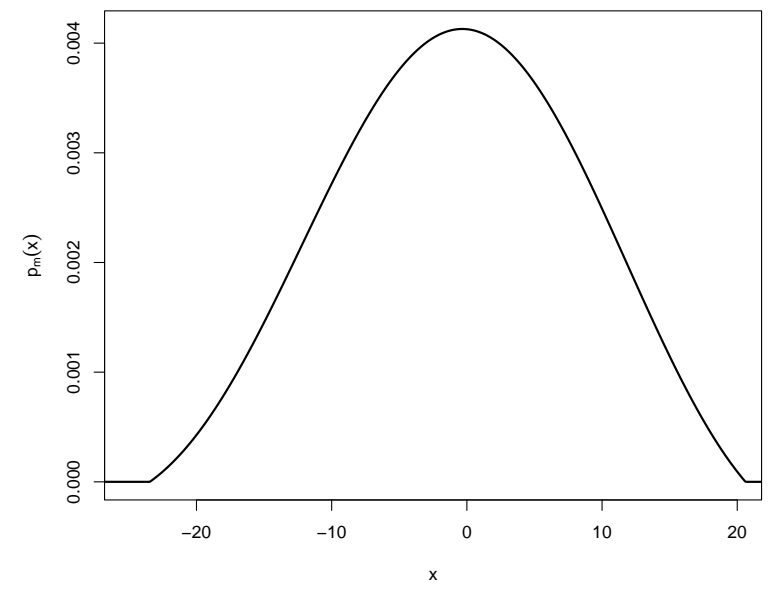

Figure 3: Density $p_{n-1}(x)$ calibrated using terminal caplet volas $\sigma_{T_{n-1}}^{K}$ 

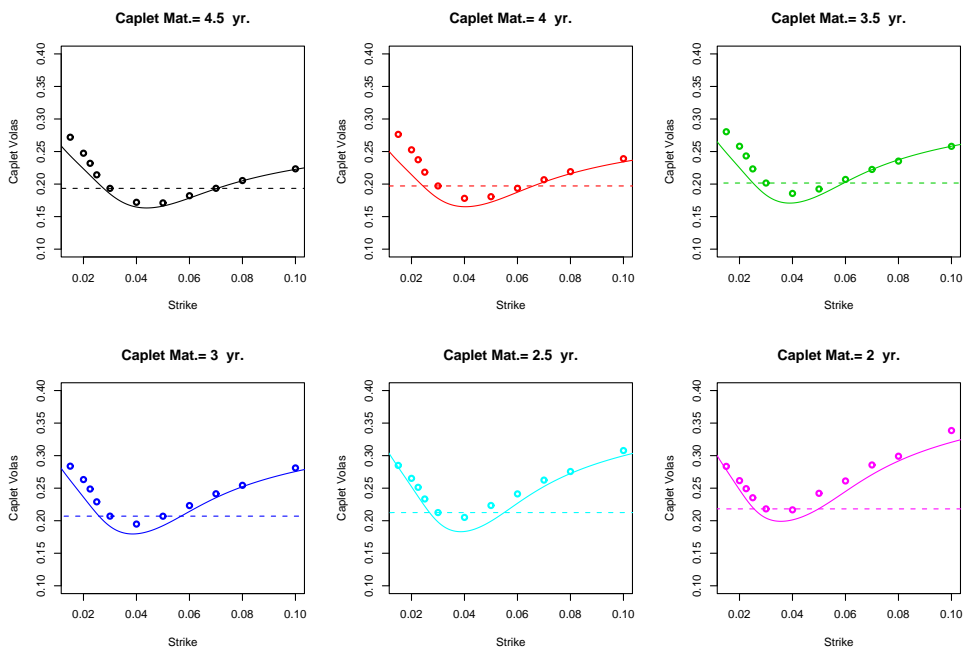

Figure 4: Caplet volas from the calibrated model (solid lines), original caplets volas $\sigma_{T}^{K}$ (points) and ATM caplet volas $\sigma_{T}^{A T M}$ (dashed lines) for different caplet maturities $T$. 

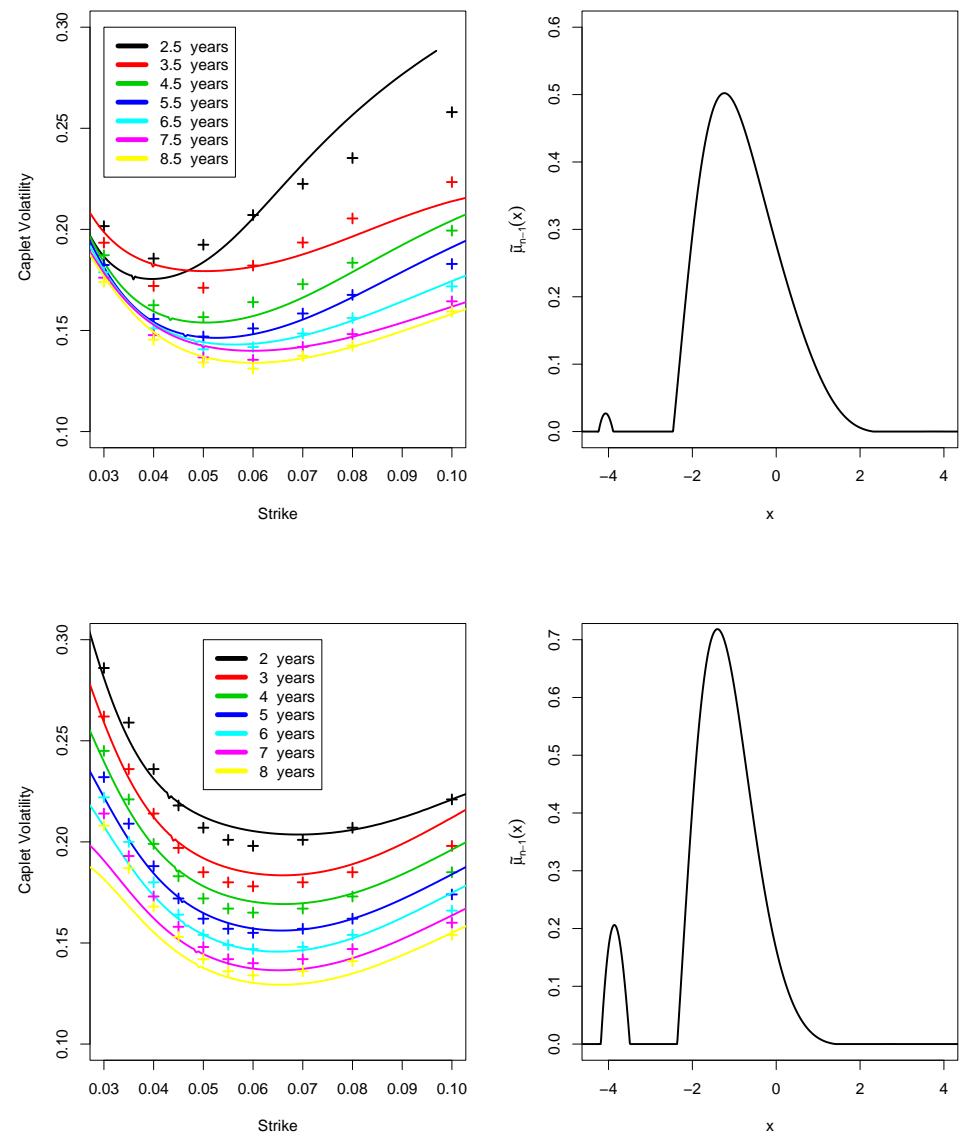

Figure 5: The comparison of estimation results for two data sets 11.01.2004 (top) and 22.10.08 (bottom). Left-hand side: caplet volas from calibrated model (solid lines), original caplets volas $\sigma_{T}^{K}$ (crosses) for different caplet maturities $T$; Right-hand side: estimated densities $\widetilde{\mu}_{n-1}^{+}\left(x, U^{*}\right)$. 